\title{
Novel improved fractional operators and their scientific applications
}

\author{
Abd-Allah Hyder ${ }^{1,2 *}$ and M.A. Barakat ${ }^{3,4}$
}

${ }^{*}$ Correspondence:
abahahmed@kku.edu.sa
'Department of Mathematics,
College of Science, King Khalid
University, P.O. Box 9004,61413,
Abha, Saudi Arabia
${ }^{2}$ Department of Engineering
Mathematics and Physics, Faculty of
Engineering, Al-Azhar University,
11371, Cairo, Egypt
Full list of author information is
available at the end of the article

available at the end of the article

\begin{abstract}
The motivation of this research is to introduce some new fractional operators called "the improved fractional (IF) operators". The originality of these fractional operators comes from the fact that they repeat the method on general forms of conformable integration and differentiation rather than on the traditional ones. Hence the convolution kernels correlating with the IF operators are served in conformable abstract forms. This extends the scientific application scope of their fractional calculus. Also, some results are acquired to guarantee that the IF operators have advantages analogous to the familiar fractional integral and differential operators. To unveil the inverse and composition properties of the IF operators, certain function spaces with their characterizations are presented and analyzed. Moreover, it is remarkable that the IF operators generalize some fractional and conformable operators proposed in abundant preceding works. As scientific applications, the resistor-capacitor electrical circuits are analyzed under some IF operators. In the case of constant and periodic sources, this results in novel voltage forms. In addition, the overall influence of the IF operators on voltage behavior is graphically simulated for certain selected fractional and conformable parameter values. From the standpoint of computation, the usage of new IF operators is not limited to electrical circuits; they could also be useful in solving scientific or engineering problems.
\end{abstract}

MSC: 26A33; 34A08; 34B30

Keywords: Fractional operators; Conformable calculus; Repeated integrals; Electrical circuits

\section{Introduction}

The traditional notions of derivatives and integrals are extended from integer orders to positive real or complex orders in fractional calculus $[2,4,5,29,48,53]$. Recently, abundant mathematicians and physicists employed fractional calculus to model intricate media and multiscale aspects in naturalistic phenomena $[3,6-9,16,35,43,52,54]$. Therefore fractional differential paradigms are extensively proposed to treat multiple problems in different fields, from control theory to electrical circuits [10, 20, 41, 46], from economics to activity of stock markets [50,51], from fluid mechanics to behavior of viscoelastic materials [37, 42], from signal processing to stochastic processes [11, 32, 38, 40], and others.

(c) The Author(s) 2021. This article is licensed under a Creative Commons Attribution 4.0 International License, which permits use sharing, adaptation, distribution and reproduction in any medium or format, as long as you give appropriate credit to the original author(s) and the source, provide a link to the Creative Commons licence, and indicate if changes were made. The images or other third party material in this article are included in the article's Creative Commons licence, unless indicated otherwise in a credit line to the material. If material is not included in the article's Creative Commons licence and your intended use is not permitted by statutory regulation or exceeds the permitted use, you will need to obtain permission directly from the copyright holder. To view a copy of this licence, visit http://creativecommons.org/licenses/by/4.0/. 
For compatibility with many scientific problems, there an assortment of fractional operator concepts is proposed. The most spreading concepts are the Riemann-Liouville and Caputo operators $[2,44]$. The Riemann-Liouville operator is defined via the Volterra integral, so that it has an integrative nature along with a convolution kernel given as a power function. This shows why it is chosen to treat many applied troubles [14, 21, 49, 52]. However, the literature did not stop at the power kernel. There are nonpower kernels, such as the exponential kernel [13] and log-normal kernel [36], which are quite decent in various approaches. Accordingly, the construction of new fractional operators is still open. Indeed, we can change the convolution kernels by more suitable forms to meet different questions.

The Riemann-Liouville operator and its variations are obviously integrative, giving them nonlocal properties. From a natural standpoint, if the action of delay is insignificant, then the nonlocality becomes a source of discomfort. So modeling such intricate phenomena needs local differential operators, which are more exact than the traditional Newton-Leibniz differential operator. In 2014, a local conformable derivative (LCD) and its associated integral was suggested by Khalil et al. [34]. From that moment, the LCD caught the attention of many authors, because it is local and elegantly covers all properties of the Newton-Leibniz derivative. Consequently, the LCD has been frequently employed to treat different development of wave equations in wide practical scopes; for instance, see $[15,17,22-24,28,45,47]$. In the Riemann-Liouville and Caputo settings, Jarad et al. [34]also created a new set of fractional operators. They developed these fractional operators using Khalil's iterative methodology on the LCD and its conformable integral. Also, the fractional operators of Jarad and his coauthors were utilized to attain exact formulas for the voltage in some electrical circuits with fractional parameters [39, 46].

Presently, it turns out that the LCD generalizes the traditional Newton-Leibniz derivative on a nonzero domain, but it has no correlation at zero. So, it is subjected to some criticism $[1,26]$. This blemish in the LCD was evaded by a new local generalized conformable derivative (LGCD), proposed by Zhao and Luo [55]. Therefore the locality and inclusiveness advantages of the LGCD give it the entitlement to occupy the place of the LCD $[26,56]$. Moreover, the LGCD provides a new application environment to gain some general results for many physical evolution models $[25,27,55,56]$.

The contribution of the present study is introducing novel sorts of fractional operators called the IF operators. The definitions of these operators are in the Riemann-Liouville and Caputo settings, but they are structured via general kinds of conformable integration and differentiation instead of the traditional ones. By a repetition procedure on the LGCD and its conformable integration we end with explicit definitions of the IF operators. From a convolution point of view, the kernels associated with these fractional operators are given as conformable abstract forms. This expands their scientific application area compared to the familiar fractional operators. Also, we show that the new IF operators have advantages analogous to those of popular fractional integral and differential operators. To clarify the relations between the IF integrals and derivatives, we introduce certain spaces and give the expansions of the IF derivatives on these spaces. An important feature of the IF operators is that they rely on double parameters so that, in modeling by these fractional operators, one of them is responsible for the memory trace and the other for the speed up or slow down of the variation rate. For particular values of these parameters, the IF operators reduce to many fractional and conformable operators proposed in numer- 
ous past researches. As workable applications, the resistor-capacitor electrical circuits are discussed in the framework of some IF operators. These applications produce new formulas of the voltage in the cases of constant and periodic sources. Moreover, some graphical simulations are provided to elucidate the overall effect of the IF operators on the voltage behavior. Here we only apply the IF operators to analyze some simple electrical circuits to demonstrate their effectiveness and their role in inducing noticeable changes in the behavior of physical phenomena. To the authors' knowledge, the applications of the new IF operators do not end with the electrical circuits, and they may have valuable applications in many practical fields.

This paper is organized as follows: Sect. 2 involves the definitions and main advantages of the novel IF operators. In Sect. 3, we introduce some specific function spaces to show the relations between the IF derivatives and integrals. Moreover, these spaces are analyzed by expanding their elements and calculating their IF derivatives. In Sect. 4, we give the definitions of the new Caputo IF derivatives and their properties. In Sect. 5, we apply some of the IF operators to resolve the resistor-capacitor electrical circuits with fractional parameters. In addition, the total impact of the IF operators on the voltage behavior is simulated graphically for some particular values of the fractional and conformable orders. Section 6 provides a conclusion.

\section{The improved fractional operators}

In this part, we introduce new fractional operators and show their advantages. We can name these operators as:

- The left improved fractional integral (LIFI).

- The right improved fractional integral (RIFI).

- The left improved fractional derivative (LIFD).

- The right improved fractional derivative (RIFD).

Also, we show that the LIFI, RIFI, LIFD, and RIFD are inclusive and cover many fractional and conformable operators proposed in multiple past works. Moreover, we provide some advantages of the LIFI, RIFI, LIFD, and RIFD, which may be employed when applying them.

\subsection{Recognizing the LIFI, RIFI, LIFD, and RIFD}

Zhao et al. [55, 56] introduced the concepts of the local generalized conformable derivative and integral (LGCD and LGCI) via the so called "conformable functions". In this portion, we start our contribution by extending their concepts to the new left and right LGCDs and LGCIs. Consequently, by iterating the left and right LGCIs $m$ times $(m \in \mathbb{N})$ and replacing the number $m$ by an arbitrary complex number, we end with the precise definitions of the LIFI, RIFI, LIFD, and RIFD.

Definition $1([55,56])$ Let $\mathcal{T}=\mathbb{R}_{+} \times(0,1]$. We denote by $\Sigma$ the set of all continuous functions from $\mathcal{T}$ into $\mathbb{R}$ such that for all $\omega \in \Sigma$ :

(1) $\omega(\vartheta, 1)=1$ for all $\vartheta \in \mathbb{R}_{+}$,

(2) $\omega(\vartheta, \rho) \neq 0$ for all $(\vartheta, \rho) \in \mathcal{T}$,

(3) $\omega\left(\cdot, \rho_{1}\right) \neq \omega\left(\cdot, \rho_{2}\right)$ for all $\rho_{1}, \rho_{2} \in(0,1]$ such that $\rho_{1} \neq \rho_{2}$.

Also, we define $\bar{\Sigma}$ as the union $\Sigma \cup\{\omega(\vartheta, \rho)=1\}$ and call any function $\omega \in \bar{\Sigma}$ a binary conformable function. 
The precise definitions of the left and right LGCDs are stated as follows.

Definition 2 Let $\omega \in \bar{\Sigma}$, and let $\xi:[\mu, \infty) \rightarrow \mathbb{R}$ be a function with $\mu \geq 0$. The left LGCD of $\xi$ of order $\rho \in(0,1]$ starting from $\mu$ is defined as

$$
D_{\mu, \omega}^{\rho}(\xi(\vartheta))=\lim _{p \rightarrow 0} \frac{\xi(\vartheta+p \omega(\vartheta-\mu, \rho))-\xi(\vartheta)}{p} .
$$

The right LGCD of $\xi$ of order $\rho \in(0,1]$ terminating at $v$ is defined as

$$
D_{\omega, v}^{\rho}(\xi(\vartheta))=\lim _{p \rightarrow 0} \frac{\xi(\vartheta+p \omega(v-\vartheta, \rho))-\xi(\vartheta)}{p} .
$$

Moreover, if $\xi$ is differentiable, then

$$
D_{\mu, \omega}^{\rho}(\xi(\vartheta))=\omega(\vartheta-\mu, \rho) \frac{d \xi}{d \vartheta} \quad \text { and } \quad D_{\omega, \nu}^{\rho}(\xi(\vartheta))=\omega(\nu-\vartheta, \rho) \frac{d \xi}{d \vartheta} .
$$

The appropriate integrals can be set up as the next forms.

Definition 3 Let $\omega \in \bar{\Sigma}$, and let $\xi:[\mu, \infty) \rightarrow \mathbb{R}$ be a function with $\mu \geq 0$. The left LGCI of $\xi$ of order $\rho \in(0,1]$ starting from $\mu$ is defined as

$$
I_{\mu, \omega}^{\rho}(\xi(\vartheta))=\int_{\mu}^{\vartheta} \frac{\xi(\tau)}{\omega(\tau-\mu, \rho)} d \tau
$$

and the right LGCI of $\xi$ of order $\rho \in(0,1]$ terminating at $v$ is defined as

$$
I_{\omega, v}^{\rho}(\xi(\vartheta))=\int_{\vartheta}^{v} \frac{\xi(\tau)}{\omega(\nu-\tau, \rho)} d \tau .
$$

The following lemma provides the some inverse attributes of the left and right LGCDs and LGCIs.

Lemma 1 Suppose $\xi:[\mu, \infty) \rightarrow \mathbb{R}$ is continuous and $\rho \in(0,1]$. Then

(i) $D_{\mu, \omega}^{\rho}\left(I_{\mu, \omega}^{\rho}(\xi(\vartheta))\right)=\xi(\vartheta)$

(ii) $I_{\omega, v}^{\rho}\left(D_{\omega, v}^{\rho}(\xi(\vartheta))\right)=\xi(\vartheta)$.

The proof of Lemma 1 can be straightaway gained from Eqs. (3), (4), and (5).

For the higher-order left and right LGCDs, we give the following:

Definition 4 Let $\rho \in(m, m+1], m \in \mathbb{N}$, and $\omega \in \bar{\Sigma}$, and let $\xi$ be an $m$-differentiable function from $[\mu, \infty)$ into $\mathbb{R}$. The left LGCD of order $\rho$ starting from $\mu \geq 0$ is defined as

$$
\mathbf{D}_{\mu, \omega}^{\rho}(\xi(\vartheta))=D_{\mu, \omega}^{\rho-m}\left(\xi^{(m)}(\vartheta)\right)=\lim _{p \rightarrow 0} \frac{\xi^{(m)}(\vartheta+p \omega(\vartheta-\mu, \rho-m))-\xi^{(m)}(\vartheta)}{p} .
$$

The right LGCD of $\xi$ of order $\rho$ terminating at $v$ is defined as

$$
\mathbf{D}_{\omega, v}^{\rho}(\xi(\vartheta))=D_{\omega, v}^{\rho-m}\left(\xi^{(m)}(\vartheta)\right)=\lim _{p \rightarrow 0} \frac{\xi^{(m)}(\vartheta+p \omega(\nu-\vartheta, \rho-m))-\xi^{(m)}(\vartheta)}{p},
$$

provided that the limits in (6) and (7) exist. 
By iterating the left LGCI (4) $m$ times we end with the following result:

$$
\begin{aligned}
\mathbf{I}_{\mu, \omega}^{m, \rho}(\xi(\vartheta)) & =\int_{\mu}^{\vartheta} \frac{d \tau_{1}}{\omega\left(\tau_{1}-\mu, \rho\right)} \int_{\mu}^{\tau_{1}} \frac{d \tau_{2}}{\omega\left(\tau_{2}-\mu, \rho\right)} \cdots \int_{\mu}^{\tau_{m-1}} \frac{\xi\left(\tau_{m}\right) d \tau_{m}}{\omega\left(\tau_{m}-\mu, \rho\right)} \\
& =\frac{1}{\Gamma(m)} \int_{\mu}^{\vartheta} h^{m-1}(\vartheta-\mu, \tau-\mu, \rho) \frac{\xi(\tau)}{\omega(\tau-\mu, \rho)} d \tau,
\end{aligned}
$$

where $\Gamma(m)$ is the gamma function, and

$$
h(\vartheta, \tau, \rho)=\int_{\tau}^{\vartheta} \frac{d u}{\omega(u, \rho)}
$$

On replacing the natural number $m$ by a complex number, we define the LIFI and RIFI as follows.

Definition 5 Let $\sigma \in \mathbb{C}$ with $\operatorname{Re}(\sigma)>0$. The LIFI is defined as

$$
\mathbb{I}_{\mu, \omega}^{\sigma, \rho}(\xi(\vartheta))=\frac{1}{\Gamma(\sigma)} \int_{\mu}^{\vartheta} h^{\sigma-1}(\vartheta-\mu, \tau-\mu, \rho) \frac{\xi(\tau)}{\omega(\tau-\mu, \rho)} d \tau,
$$

and the RIFI is defined as

$$
\mathbb{I}_{\omega, \nu}^{\sigma, \rho}(\xi(\vartheta))=\frac{1}{\Gamma(\sigma)} \int_{\vartheta}^{v} h^{\sigma-1}(\nu-\vartheta, v-\tau, \rho) \frac{\xi(\tau)}{\omega(\nu-\tau, \rho)} d \tau
$$

$\operatorname{Remark} 1$ If $(Z \xi)(\vartheta)=\xi(\mu+\nu-\vartheta)$, then $\mathbb{I}_{\mu, \omega}^{\sigma, \rho}((Z \xi)(\vartheta))=\mathbb{I}_{\omega, \nu}^{\sigma, \rho}(\xi(\vartheta))$. Indeed,

$$
\begin{aligned}
\mathbb{I}_{\mu, \omega}^{\sigma, \rho}((Z \xi)(\vartheta)) & =\frac{1}{\Gamma(\sigma)} \int_{\mu}^{\mu+\nu-\vartheta} h^{\sigma-1}(\nu-\vartheta, \tau-\mu, \rho) \frac{\xi(\mu+\nu-\tau)}{\omega(\tau-\mu, \rho)} d \tau \\
& =\frac{1}{\Gamma(\sigma)} \int_{\vartheta}^{v} h^{\sigma-1}(\nu-\vartheta, \nu-\tau, \rho) \frac{\xi(\tau)}{\omega(\nu-\tau, \rho)} d \tau \\
& =\mathbb{I}_{\omega, \nu}^{\sigma, \rho}(\xi(\vartheta)) .
\end{aligned}
$$

Remark 2 The LIFI and RIFI include some fractional integral operators defined in many previous works. The following items illustrate this fact.

(1) If $\omega(\vartheta, \rho)=\vartheta^{1-\rho}$, then $h(\vartheta, \tau, \rho)=\frac{\vartheta^{\rho}-\tau^{\rho}}{\rho}$ and, respectively, the LIFI and RIFI in (10) and (11) coincide with the left and right fractional integrals defined by Jarad et al. [31].

(2) If $\omega(\vartheta, \rho)=\vartheta^{1-\rho}, \mu=v=0$, then the LIFI and RIFI in (10) and (11) coincide with the generalized fractional left and right integrals defined by Katugampola in [33], respectively.

(3) If $\omega(\vartheta, \rho)=\vartheta^{1-\rho}, \rho=1, \mu=v=0$, then $h(\vartheta, \tau, \rho)=\vartheta-\tau$, and, respectively, the LIFI and RIFI in (10) and (11) coincide with the fractional left and right integrals of Riemann-Liouville defined in $[2,44]$.

(4) If $\omega(\vartheta, \rho)=\vartheta^{1-\rho}, \mu=0, \rho \rightarrow 0$, then $h(\vartheta, \tau, \rho) \rightarrow \ln (\vartheta)-\ln (\tau)$, and the LIFI and RIFI in (10) and (11) coincide with the fractional left and right integrals of Hadamard [36], respectively. 
Now we can present the definitions of the LIFD and RIFD.

Definition 6 Let $\sigma \in \mathbb{C}$ with $\operatorname{Re}(\sigma)>0$. In the Riemann-Liouville setting the LIFD of order $\sigma$ is defined by

$$
\begin{aligned}
\mathbb{D}_{\mu, \omega}^{\sigma, \rho}(\xi(\vartheta)) & =D_{\mu, \omega}^{m, \rho}\left(\mathbb{I}_{\mu, \omega}^{m-\sigma, \rho}(\xi(\vartheta))\right) \\
& =\frac{1}{\Gamma(m-\sigma)} D_{\mu, \omega}^{m, \rho}\left(\int_{\mu}^{\vartheta} h^{m-\sigma-1}(\vartheta-\mu, \tau-\mu, \rho) \frac{\xi(\tau)}{\omega(\tau-\mu, \rho)} d \tau\right),
\end{aligned}
$$

and the RIFD of order $\sigma$ is defined by

$$
\begin{aligned}
\mathbb{D}_{\omega, \nu}^{\sigma, \rho}(\xi(\vartheta)) & =(-1)^{m} D_{\omega, \nu}^{m, \rho}\left(\mathbb{I}_{\omega, \nu}^{m-\sigma, \rho}(\xi(\vartheta))\right) \\
& =\frac{(-1)^{m}}{\Gamma(m-\sigma)} D_{\omega, \nu}^{m, \rho}\left(\int_{\vartheta}^{v} h^{m-\sigma-1}(v-\vartheta, v-\tau, \rho) \frac{\xi(\tau)}{\omega(v-\tau, \rho)} d \tau\right),
\end{aligned}
$$

where

$$
m=[\operatorname{Re}(\sigma)]+1, \quad D_{\mu, \omega}^{m, \rho}=\underbrace{D_{\mu, \omega}^{\rho} D_{\mu, \omega}^{\rho} \cdots D_{\mu, \omega}^{\rho}}_{m \text { times }}, \quad D_{\omega, \nu}^{m, \rho}=\underbrace{D_{\omega, \nu}^{\rho} D_{\omega, \nu}^{\rho} \cdots D_{\omega, \nu}^{\rho}}_{m \text { times }},
$$

and $D_{\mu, \omega}^{\rho}$ and $D_{\omega, v}^{\rho}$ are the left and right LGCDs defined in (1) and (2), respectively.

Remark 3 The LIFD and RIFD cover some fractional differential operators proposed in diverse past works. The following cases explain this fact.

(1) If $\omega(\vartheta, \rho)=\vartheta^{1-\rho}$, then $h(\vartheta, \tau, \rho)=\frac{\vartheta^{\rho}-\tau^{\rho}}{\rho}, D_{\mu, \omega}^{\rho}(\xi(\vartheta))=(\vartheta-\mu)^{1-\rho} \frac{d \xi}{d \vartheta}$, and $D_{\omega, v}^{\rho}(\xi(\vartheta))=(v-\vartheta)^{1-\rho} \frac{d \xi}{d \vartheta}$. Hence the LIFD and RIFD in (13)-(15) match with the left and right fractional derivatives defined by Jarad et al. [31], respectively.

(2) If $\omega(\vartheta, \rho)=\vartheta^{1-\rho}$ and $\mu=v=0$, theb the LIFD and RIFD in (13)-(15) match with the generalized fractional left and right derivatives defined by Katugampola [33], respectively.

(3) If $\omega(\vartheta, \rho)=\vartheta^{1-\rho}, \rho=1$, and $\mu=v=0$, then $h(\vartheta, \tau, \rho)=\vartheta-\tau$ and $D_{\mu, \omega}^{\rho}(\xi(\vartheta))=D_{\omega, v}^{\rho}(\xi(\vartheta))=\frac{d \xi}{d \vartheta}$. Hence, respectively, the LIFD and RIFD in (13)-(15) match with the fractional left and right derivatives of Riemann-Liouville defined in $[2,44]$.

(4) If $\omega(\vartheta, \rho)=\vartheta^{1-\rho}, \mu=0$, and $\rho \rightarrow 0$, then $h(\vartheta, \tau, \rho) \rightarrow \ln (\vartheta)-\ln (\tau)$, $D_{\mu, \omega}^{\rho}(\xi(\vartheta)) \rightarrow \vartheta \frac{d \xi}{d \vartheta}, D_{\omega, v}^{\rho}(\xi(\vartheta)) \rightarrow-\vartheta \frac{d \xi}{d \vartheta}$. Hence, respectively, the LIFD and RIFD in (13)-(15) match with the fractional left and right derivatives of Hadamard [36].

\subsection{Some advantages of the LIFI, RIFI, LIFD, and RIFD}

We provide some results to guarantee that the LIFI, RIFI, LIFD, and RIFD have advantages analogous to those of the familiar fractional integral and differential operators.

Lemma 2 Let $\operatorname{Re}\left(\sigma_{1}\right)>0$ and $\operatorname{Re}\left(\sigma_{2}\right)>0$. Then:

(i) $\mathbb{I}_{\mu, \omega}^{\sigma_{1}, \rho}\left(\mathbb{I}_{\mu, \omega}^{\sigma_{2}, \rho}(\xi(\vartheta))\right)=\mathbb{I}_{\mu, \omega}^{\sigma_{1}+\sigma_{2}, \rho}(\xi(\vartheta))$,

(ii) $\mathbb{I}_{\omega, \nu}^{\sigma_{1}, \rho}\left(\mathbb{I}_{\omega, \nu}^{\sigma_{2}, \rho}(\xi(\vartheta))\right)=\mathbb{I}_{\omega, v}^{\sigma_{1}+\sigma_{2}, \rho}(\xi(\vartheta))$. 
Proof By using Eq. (10) and changing the order of integration we have

$$
\begin{aligned}
\mathbb{I}_{\mu, \omega}^{\sigma_{1}, \rho}\left(\mathbb{I}_{\mu, \omega}^{\sigma_{2}, \rho}(\xi(\vartheta))=\right. & \frac{1}{\Gamma\left(\sigma_{1}\right) \Gamma\left(\sigma_{2}\right)} \int_{\mu}^{\vartheta} \int_{\mu}^{\tau_{1}} h^{\sigma_{1}-1}\left(\vartheta-\mu, \tau_{1}-\mu, \rho\right) h^{\sigma_{2}-1}\left(\tau_{1}-\mu, \tau_{2}-\mu, \rho\right) \\
& \times \frac{\xi\left(\tau_{2}\right) d \tau_{2}}{\omega\left(\tau_{2}-\mu, \rho\right)} \frac{d \tau_{1}}{\omega\left(\tau_{1}-\mu, \rho\right)} \\
= & \frac{1}{\Gamma\left(\sigma_{1}\right) \Gamma\left(\sigma_{2}\right)} \int_{\mu}^{\vartheta} \int_{\tau_{2}}^{\vartheta} h^{\sigma_{1}-1}\left(\vartheta-\mu, \tau_{1}-\mu, \rho\right) h^{\sigma_{2}-1}\left(\tau_{1}-\mu, \tau_{2}-\mu, \rho\right) \\
& \times \frac{d \tau_{1}}{\omega\left(\tau_{1}-\mu, \rho\right)} \frac{\xi\left(\tau_{2}\right) d \tau_{2}}{\omega\left(\tau_{2}-\mu, \rho\right)} \\
= & \frac{1}{\Gamma\left(\sigma_{1}\right) \Gamma\left(\sigma_{2}\right)}\left(\int_{0}^{1}(1-\varrho)^{\sigma_{1}-1} \varrho^{\sigma_{2}-1} d \varrho\right) \\
& \times \int_{\mu}^{\vartheta} h^{\sigma_{1}+\sigma_{2}-1}\left(\vartheta-\mu, \tau_{2}-\mu, \rho\right) \frac{\xi\left(\tau_{2}\right) d \tau_{2}}{\omega\left(\tau_{2}-\mu, \rho\right)} \\
= & \frac{1}{\Gamma\left(\sigma_{1}+\sigma_{2}\right)} \int_{\mu}^{\vartheta} h^{\sigma_{1}+\sigma_{2}-1}\left(\vartheta-\mu, \tau_{2}-\mu, \rho\right) \frac{\xi\left(\tau_{2}\right) d \tau_{2}}{\omega\left(\tau_{2}-\mu, \rho\right)} \\
= & \mathbb{I}_{\mu, \omega}^{\sigma_{1}+\sigma_{2}, \rho}(\xi(\vartheta)) .
\end{aligned}
$$

Here we have employed the variable transformation $\varrho=\frac{h\left(\tau_{1}-\mu, \tau_{2}-\mu, \rho\right)}{h\left(\vartheta-\mu, \tau_{2}-\mu, \rho\right)}$, the definition of the renowned beta function $B\left(\sigma_{1}, \sigma_{2}\right)=\int_{0}^{1}(1-\varrho)^{\sigma_{1}-1} \varrho^{\sigma_{2}-1} d \varrho$, and the identity $B\left(\sigma_{1}, \sigma_{2}\right)=$ $\frac{\Gamma\left(\sigma_{1}\right) \Gamma\left(\sigma_{2}\right)}{\Gamma\left(\sigma_{1}+\sigma_{2}\right)}$. This proves (i), and (ii) can be proved similarly.

Remark 4 If If $\omega(\vartheta, \rho)=\vartheta^{1-\rho}$, then $h(\vartheta, \tau, \rho)=\frac{\vartheta^{\rho}-\tau^{\rho}}{\rho}$, and Lemma 2 reduces to the result obtained by Jarad et al. [31, Theorem 2.1].

Lemma 3 Let $\sigma, \delta \in \mathbb{C}$ with $\operatorname{Re}(\sigma)>0$ and $\operatorname{Re}(\delta)>0$. Then:

(i) $\mathbb{I}_{\mu, \omega}^{\sigma, \rho}\left(h^{\delta-1}(\vartheta-\mu, 0, \rho)\right)=\frac{\Gamma(\delta)}{\Gamma(\sigma+\delta)} h^{\sigma+\delta-1}(\vartheta-\mu, 0, \rho)$,

(ii) $\mathbb{I}_{\omega, \nu}^{\sigma, \rho}\left(h^{\delta-1}(v-\vartheta, 0, \rho)\right)=\frac{\Gamma(\delta)}{\Gamma(\sigma+\delta)} h^{\sigma+\delta-1}(v-\vartheta, 0, \rho)$,

where $h$ is given in Eq. (9).

Proof According to Definition 5, we have

$$
\mathbb{I}_{\mu, \omega}^{\sigma, \rho}\left(h^{\delta-1}(\vartheta-\mu, 0, \rho)\right)=\frac{1}{\Gamma(\sigma)} \int_{\mu}^{\vartheta} h^{\sigma-1}(\vartheta-\mu, \tau-\mu, \rho) \frac{h^{\delta-1}(\tau-\mu, 0, \rho)}{\omega(\tau-\mu, \rho)} d \tau .
$$

Applying the variable transformation $\varrho=\frac{h(\tau-\mu, 0, \rho)}{h(\vartheta-\mu, 0, \rho)}$, we get

$$
\begin{aligned}
\mathbb{I}_{\mu, \omega}^{\sigma, \rho}\left(h^{\delta-1}(\vartheta-\mu, 0, \rho)\right)= & \frac{1}{\Gamma(\sigma)} \int_{0}^{1}[h(\vartheta-\mu, 0, \rho)-\varrho h(\vartheta-\mu, 0, \rho)]^{\sigma-1} \\
& \times \varrho^{\delta-1} h^{\delta}(\vartheta-\mu, 0, \rho) d \varrho \\
= & \frac{h^{\sigma+\delta-1}(\vartheta-\mu, 0, \rho)}{\Gamma(\sigma)} \int_{0}^{1}(1-\varrho)^{\sigma_{1}-1} \varrho^{\sigma_{2}-1} d \varrho \\
= & \frac{\Gamma(\delta)}{\Gamma(\delta+\sigma)} h^{\sigma+\delta-1}(\vartheta-\mu, 0, \rho) .
\end{aligned}
$$

Hence (i) is proved, and (ii) can be proved in a similar way. 
Remark 5 If If $\omega(\vartheta, \rho)=\vartheta^{1-\rho}$, then $h(\vartheta, 0, \rho)=\frac{\vartheta^{\rho}}{\rho}$, and Lemma 3 covers the result obtained by Jarad et al. [31, Lemma 2.2].

Lemma 4 If $\operatorname{Re}(\delta-\sigma)>0$ for $\delta, \sigma \in \mathbb{C}$, then:

(i) $\mathbb{D}_{\mu, \omega}^{\sigma, \rho}\left(h^{\delta-1}(\vartheta-\mu, 0, \rho)\right)=\frac{\Gamma(\delta)}{\Gamma(\delta-\sigma)} h^{\delta-\sigma-1}(\vartheta-\mu, 0, \rho)$,

(ii) $\mathbb{D}_{\omega, \nu}^{\sigma, \rho}\left(h^{\delta-1}(v-\vartheta, 0, \rho)\right)=\frac{\Gamma(\delta)}{\Gamma(\delta-\sigma)} h^{\delta-\sigma-1}(v-\vartheta, 0, \rho)$,

where h is given in Eq. (9).

Proof According to Definition 6 and Lemma 3, we have

$$
\begin{aligned}
\mathbb{D}_{\mu, \omega}^{\sigma, \rho}\left(h^{\delta-1}(\vartheta-\mu, 0, \rho)\right) & =D_{\mu, \omega}^{m, \rho}\left(\mathbb{I}_{\mu, \omega}^{m-\sigma, \rho}\left(h^{\delta-1}(\vartheta-\mu, 0, \rho)\right)\right) \\
& =\frac{\Gamma(\delta)}{\Gamma(\delta-\sigma+m)} D_{\mu, \omega}^{m, \rho}\left(h^{\delta-\sigma+m-1}(\vartheta-\mu, 0, \rho)\right) \\
& =\frac{\Gamma(\delta)}{\Gamma(\delta-\sigma)} h^{\delta-\sigma-1}(\vartheta-\mu, 0, \rho) .
\end{aligned}
$$

Hence (i) is proved, and (ii) can be proved in a similar way.

Remark 6 If If $\omega(\vartheta, \rho)=\vartheta^{1-\rho}$, then $h(\vartheta, 0, \rho)=\frac{\vartheta^{\rho}}{\rho}$, and Lemma 4 reduces to the result proved by Jarad et al. [31, Lemma 2.3].

The following lemma assists in computing the generalized conformable derivatives of the LIFI and RIFI.

Lemma 5 Let $\sigma \in \mathbb{C}$ and $m \in \mathbb{N}$ with $\operatorname{Re}(\sigma)>m$. Then:

(i) $D_{\mu, \omega}^{m, \rho}\left(\mathbb{I}_{\mu, \omega}^{\sigma, \rho}(\xi(\vartheta))\right)=\mathbb{I}_{\mu, \omega}^{\sigma-m, \rho}(\xi(\vartheta))$,

(ii) $D_{\omega, v}^{m, \rho}\left(\mathbb{I}_{\omega, \nu}^{\sigma, \rho}(\xi(\vartheta))\right)=\mathbb{I}_{\omega, \nu}^{\sigma-m, \rho}(\xi(\vartheta))$.

Proof We have

$$
\begin{aligned}
D_{\mu, \omega}^{m, \rho}\left(\mathbb{I}_{\mu, \omega}^{\sigma, \rho}(\xi(\vartheta))\right)= & D_{\mu, \omega}^{m, \rho}\left[\frac{1}{\Gamma(\sigma)} \int_{\mu}^{\vartheta} h^{\sigma-1}(\vartheta-\mu, \tau-\mu, \rho) \frac{\xi(\tau)}{\omega(\tau-\mu, \rho)} d \tau\right] \\
= & D_{\mu, \omega}^{m-1, \rho}\left[\frac{1}{\Gamma(\sigma-1)} \int_{\mu}^{\vartheta} h^{\sigma-2}(\vartheta-\mu, \tau-\mu, \rho) \frac{\xi(\tau)}{\omega(\tau-\mu, \rho)} d \tau\right] \\
= & D_{\mu, \omega}^{m-2, \rho}\left[\frac{1}{\Gamma(\sigma-2)} \int_{\mu}^{\vartheta} h^{\sigma-3}(\vartheta-\mu, \tau-\mu, \rho) \frac{\xi(\tau)}{\omega(\tau-\mu, \rho)} d \tau\right] \\
& \vdots \\
= & \frac{1}{\Gamma(\sigma-m)} \int_{\mu}^{\vartheta} h^{\sigma-m-1}(\vartheta-\mu, \tau-\mu, \rho) \frac{\xi(\tau)}{\omega(\tau-\mu, \rho)} d \tau \\
= & \mathbb{I}_{\mu, \omega}^{\sigma-m, \rho}(\xi(\vartheta)) .
\end{aligned}
$$

Now we can show the inverse advantages for different orders of the LIFD, RIFD, LIFI and RIFI. The inverse advantages with the same order need some specific function spaces, which will be discussed in the following section. 
Theorem 1 For $\operatorname{Re}\left(\sigma_{1}\right)<\operatorname{Re}\left(\sigma_{2}\right)$, we have:

(i) $\mathbb{D}_{\mu, \omega}^{\sigma_{1}, \rho}\left(\mathbb{I}_{\mu, \omega}^{\sigma_{2}, \rho}(\xi(\vartheta))\right)=\mathbb{I}_{\mu, \omega}^{\sigma_{2}-\sigma_{1}, \rho}(\xi(\vartheta))$,

(ii) $\mathbb{D}_{\omega, \nu}^{\sigma_{1}, \rho}\left(\mathbb{I}_{\omega, \nu}^{\sigma_{2}, \rho}(\xi(\vartheta))\right)=\mathbb{I}_{\omega, \nu}^{\sigma_{2}-\sigma_{1}, \rho}(\xi(\vartheta))$.

Proof From Lemmas 2 and 5 we have

$$
\begin{aligned}
\mathbb{D}_{\mu, \omega}^{\sigma_{1}, \rho}\left(\mathbb{I}_{\mu, \omega}^{\sigma_{2}, \rho}(\xi(\vartheta))\right) & =D_{\mu, \omega}^{m, \rho}\left(\mathbb{I}_{\mu, \omega}^{m-\sigma_{1}, \rho}\left(\mathbb{I}_{\mu, \omega}^{\sigma_{2}, \rho}(\xi(\vartheta))\right)\right) \\
& =D_{\mu, \omega}^{m, \rho}\left(\mathbb{I}_{\mu, \omega}^{\sigma_{2}-\sigma_{1}+m, \rho}(\xi(\vartheta))\right) \\
& =\mathbb{I}_{\mu, \omega}^{\sigma_{2}-\sigma_{1}, \rho}(\xi(\vartheta)) .
\end{aligned}
$$

So (i) is proved, and (ii) can be proved similarly.

Theorem 2 For $\operatorname{Re}\left(\sigma_{1}\right)>0$ and $\operatorname{Re}\left(\sigma_{1}\right)<0$, we have:

(i) $\mathbb{I}_{\mu, \omega}^{\sigma_{1}, \rho}\left(\mathbb{D}_{\mu, \omega}^{\sigma_{2}, \rho}(\xi(\vartheta))\right)=\mathbb{I}_{\mu, \omega}^{\sigma_{1}-\sigma_{2}, \rho}(\xi(\vartheta))$,

(ii) $\mathbb{I}_{\omega, \nu}^{\sigma_{1}, \rho}\left(\mathbb{D}_{\omega, \nu}^{\sigma_{2}, \rho}(\xi(\vartheta))\right)=\mathbb{I}_{\omega, \nu}^{\sigma_{1}-\sigma_{2}, \rho}(\xi(\vartheta))$.

Proof According to Lemmas 2 and 5, we have

$$
\begin{aligned}
\mathbb{I}_{\mu, \omega}^{\sigma_{1}, \rho}\left(\mathbb{D}_{\mu, \omega}^{\sigma_{2}, \rho}(\xi(\vartheta))\right) & =\mathbb{I}_{\mu, \omega}^{\sigma_{1}, \rho}\left(D_{\mu, \omega}^{m, \rho}\left(\mathbb{I}_{\mu, \omega}^{m-\sigma_{2}, \rho}(\xi(\vartheta))\right)\right) \\
& =\mathbb{I}_{\mu, \omega}^{\sigma_{1}, \rho}\left(\mathbb{I}_{\mu, \omega}^{-\sigma_{2}, \rho}(\xi(\vartheta))\right) \\
& =\mathbb{I}_{\mu, \omega}^{\sigma_{1}-\sigma_{2}, \rho}(\xi(\vartheta)) .
\end{aligned}
$$

This proves (i), and the proof of (ii) can be done in a similar way.

\section{The improved fractional derivatives on the spaces $W_{\mu, \omega}^{m, \rho}$ and $W_{\omega, v}^{m, \rho}$}

We introduce certain spaces to clarify the relations between the IF integrals and derivatives. We analyze these spaces analyzed by expanding their elements and computing their IF derivatives. The obtained results of this section are needed in the next section for defining and discussing the IF derivatives in the Caputo sense.

Definition 7 Let $\rho \in(0,1]$ and $\omega \in \bar{\Sigma}$. We define

$$
I_{\mu, \omega}^{\rho}([\mu, \nu])=\left\{\xi:[\mu, \nu] \rightarrow \mathbb{R}: \xi(\vartheta)=I_{\mu, \omega}^{\rho}(f(\vartheta))+\xi(\mu), f \in N_{\rho}(\mu)\right\}
$$

and

$$
I_{\omega, \nu}^{\rho}([\mu, \nu])=\left\{\xi:[\mu, \nu] \rightarrow \mathbb{R}: \xi(\vartheta)=I_{\omega, v}^{\rho}(f(\vartheta))+\xi(\nu), f \in N_{\rho}(\nu)\right\}
$$

where

$$
\begin{aligned}
& N_{\rho}(\mu)=\left\{f:[\mu, \nu] \rightarrow \mathbb{R}: I_{\mu, \omega}^{\rho}(f(\vartheta)) \text { exists } \forall \vartheta \in[\mu, \nu]\right\}, \\
& N_{\rho}(\nu)=\left\{f:[\mu, \nu] \rightarrow \mathbb{R}: I_{\omega, \nu}^{\rho}(f(\vartheta)) \text { exists } \forall \vartheta \in[\mu, \nu]\right\},
\end{aligned}
$$

and $I_{\mu, \omega}^{\rho}$ and $I_{\omega, \nu}^{\rho}$ are the left and right LGCIs defined in Eqs. (4) and (5), respectively. 
Definition 8 For $m \in \mathbb{N}$, we define the spaces

$$
W_{\mu, \omega}^{m, \rho}([\mu, \nu])=\left\{\xi:[\mu, \nu] \rightarrow \mathbb{R}: D_{\mu, \omega}^{m-1, \rho}(\xi) \in I_{\mu, \omega}^{\rho}([\mu, \nu])\right\}
$$

and

$$
W_{\omega, \nu}^{m, \rho}([\mu, \nu])=\left\{\xi:[\mu, \nu] \rightarrow \mathbb{R}: D_{\mu, \omega}^{m-1, \rho}(\xi) \in I_{\omega, \nu}^{\rho}([\mu, \nu])\right\}
$$

where $I_{\mu, \omega}^{\rho}([\mu, \nu])$ and $I_{\omega, v}^{\rho}([\mu, \nu])$ are the spaces given in Definition 7.

\subsection{The LIFD and RIFD for functions in $W_{\mu, \omega}^{m, \rho}$ and $W_{\omega, v}^{m, \rho}$}

The following lemmas give the characterizations for the spaces $W_{\mu, \omega}^{m, \rho}([\mu, \nu])$ and $W_{\omega, v}^{m, \rho}([\mu, v])$.

Lemma 6 Let $\rho>0$. A function $\xi \in W_{\mu, \omega}^{m, \rho}([\mu, \nu])$ if and only if $\xi$ has the formal representation

$$
\begin{aligned}
\xi(\vartheta)= & \frac{1}{\Gamma(m)} \int_{\mu}^{\vartheta} h^{m-1}(\vartheta-\mu, \tau-\mu, \rho) \frac{f(\tau)}{\omega(\tau-\mu, \rho)} d \tau \\
& +\sum_{k=1}^{m-1} \frac{D_{\mu, \omega}^{k, \rho}(\xi(\mu))}{k !} h^{k}(\vartheta-\mu, 0, \rho),
\end{aligned}
$$

where $f(\vartheta)=D_{\mu, \omega}^{m, \rho}(\xi(\vartheta))$.

Proof Let $\xi \in W_{\mu, \omega}^{m, \rho}([\mu, \nu])$. Then $D_{\mu, \omega}^{m-1, \rho}(\xi) \in I_{\mu, \omega}^{\rho}([\mu, \nu])$, and hence

$$
D_{\mu, \omega}^{m-1, \rho}(\xi(\vartheta))=I_{\mu, \omega}^{\rho}(f(\vartheta))+D_{\mu, \omega}^{m-1, \rho}(\xi(\mu)) \quad \text { for some } f \in N_{\rho}(\mu) .
$$

According to Definitions 2 and 3, we get

$$
\frac{d}{d \vartheta}\left(D_{\mu, \omega}^{m-2, \rho}(\xi(\vartheta))\right)=\frac{1}{\omega(\vartheta-\mu, \rho)} \int_{\mu}^{\vartheta} \frac{f(\tau) d \tau}{\omega(\tau-\mu, \rho)}+\frac{D_{\mu, \omega}^{m-1, \rho}(\xi(\mu))}{\omega(\vartheta-\mu, \rho)} .
$$

Integrating from $\mu$ to $\vartheta$ gives

$$
\begin{aligned}
D_{\mu, \omega}^{m-2, \rho}(\xi(\vartheta))= & \int_{\mu}^{\vartheta} \frac{d \tau_{1}}{\omega\left(\tau_{1}-\mu, \rho\right)} \int_{\mu}^{\tau_{1}} \frac{f\left(\tau_{2}\right) d \tau_{2}}{\omega\left(\tau_{2}-\mu, \rho\right)} \\
& +D_{\mu, \omega}^{m-1, \rho}(\xi(\mu)) \int_{\mu}^{\vartheta} \frac{d \tau}{\omega(\tau-\mu, \rho)}+D_{\mu, \omega}^{m-2, \rho}(\xi(\mu)) .
\end{aligned}
$$

Again, by Definitions 2 and 3, integrating from $\mu$ to $\vartheta$, we get

$$
\begin{aligned}
D_{\mu, \omega}^{m-3, \rho}(\xi(\vartheta))= & \int_{\mu}^{\vartheta} \frac{d \tau_{1}}{\omega\left(\tau_{1}-\mu, \rho\right)} \int_{\mu}^{\tau_{1}} \frac{d \tau_{2}}{\omega\left(\tau_{2}-\mu, \rho\right)} \int_{\mu}^{\tau_{2}} \frac{f\left(\tau_{3}\right) d \tau_{3}}{\omega\left(\tau_{3}-\mu, \rho\right)} \\
& +D_{\mu, \omega}^{m-1, \rho}(\xi(\mu)) \int_{\mu}^{\vartheta} \frac{d \tau_{1}}{\omega\left(\tau_{1}-\mu, \rho\right)} \int_{\mu}^{\tau_{1}} \frac{d \tau_{2}}{\omega\left(\tau_{2}-\mu, \rho\right)} \\
& +D_{\mu, \omega}^{m-2, \rho}(\xi(\mu)) \int_{\mu}^{\vartheta} \frac{d \tau}{\omega(\tau-\mu, \rho)}+D_{\mu, \omega}^{m-3, \rho}(\xi(\mu)) .
\end{aligned}
$$


Repeating this procedure $m-3$ times, we have

$$
\begin{aligned}
\xi(\vartheta)= & \int_{\mu}^{\vartheta} \frac{d \tau_{1}}{\omega\left(\tau_{1}-\mu, \rho\right)} \int_{\mu}^{\tau_{1}} \frac{d \tau_{2}}{\omega\left(\tau_{2}-\mu, \rho\right)} \cdots \int_{\mu}^{\tau_{m-1}} \frac{f\left(\tau_{m}\right) d \tau_{m}}{\omega\left(\tau_{m}-\mu, \rho\right)} \\
& +D_{\mu, \omega}^{m-1, \rho}(\xi(\mu)) \int_{\mu}^{\vartheta} \frac{d \tau_{1}}{\omega\left(\tau_{1}-\mu, \rho\right)} \int_{\mu}^{\tau_{1}} \frac{d \tau_{2}}{\omega\left(\tau_{2}-\mu, \rho\right)} \cdots \int_{\mu}^{\tau_{m-2}} \frac{d \tau_{m-1}}{\omega\left(\tau_{m-1}-\mu, \rho\right)} \\
& \vdots \\
& +D_{\mu, \omega}^{1, \rho}(\xi(\mu)) \int_{\mu}^{\vartheta} \frac{d \tau}{\omega(\tau-\mu, \rho)}+\xi(\mu) .
\end{aligned}
$$

Therefore by relation (8) we have

$$
\begin{aligned}
\xi(\vartheta)= & \frac{1}{\Gamma(m)} \int_{\mu}^{\vartheta} h^{m-1}(\vartheta-\mu, \tau-\mu, \rho) \frac{f(\tau)}{\omega(\tau-\mu, \rho)} d \tau \\
& +\sum_{k=1}^{m-1} \frac{D_{\mu, \omega}^{k, \rho}(\xi(\mu))}{\Gamma(k)} \int_{\mu}^{\vartheta} \frac{h^{k-1}(\vartheta-\mu, \tau-\mu, \rho)}{\omega(\tau-\mu, \rho)} d \tau+\xi(\mu),
\end{aligned}
$$

or

$$
\begin{aligned}
\xi(\vartheta)= & \frac{1}{\Gamma(m)} \int_{\mu}^{\vartheta} h^{m-1}(\vartheta-\mu, \tau-\mu, \rho) \frac{f(\tau)}{\omega(\tau-\mu, \rho)} d \tau \\
& +\sum_{k=1}^{m-1} \frac{D_{\mu, \omega}^{k, \rho}(\xi(\mu))}{k !} h^{k}(\vartheta-\mu, 0, \rho) .
\end{aligned}
$$

Obviously, the identity $f(\vartheta)=D_{\mu, \omega}^{m, \rho}(\xi(\vartheta))$ can be obtained by taking the operator $D_{\mu, \omega}^{m, \rho}$ to the both sides of Eq. (36).

Conversely, if a function $\xi:[\mu, \nu] \rightarrow \mathbb{R}$ has the formal representation (29), then we easily conclude that $D_{\mu, \omega}^{m-1, \rho}(\xi) \in I_{\mu, \omega}^{\rho}([\mu, \nu])$ and $\xi \in W_{\mu, \omega}^{m, \rho}([\mu, \nu])$.

Lemma 7 A function $\xi \in W_{\omega, \nu}^{m, \rho}([\mu, v])$ if and only if $\xi$ has the formal representation

$$
\begin{aligned}
\xi(\vartheta)= & \frac{1}{\Gamma(m)} \int_{\vartheta}^{v} h^{m-1}(v-\vartheta, v-\tau, \rho) \frac{f(\tau)}{\omega(v-\tau, \rho)} d \tau \\
& +\sum_{k=1}^{m-1} \frac{D_{\mu, \omega}^{k, \rho}(\xi(\mu))}{k !} h^{k}(v-\vartheta, 0, \rho),
\end{aligned}
$$

where $f(\vartheta)=D_{\omega, \nu}^{m, \rho}(\xi(\vartheta))$.

Proof The proof can be driven similarly to the proof of Lemma 6.

The following theorem gives the possibility of computing the LIFD and RIFD for functions in $W_{\mu, \omega}^{m, \rho}$ and $W_{\omega, \nu}^{m, \rho}$.

Theorem 3 Let $\sigma \in \mathbb{C}$ with $\operatorname{Re}(\sigma)>0$, and let $m=[\operatorname{Re}(\sigma)]+1$. Then: 
(i) If $\xi \in W_{\mu, \omega}^{m, \rho}([\mu, \nu])$, then the LIFD of $\xi$ of order $\sigma$ exists and can be written in the form

$$
\begin{aligned}
\mathbb{D}_{\mu, \omega}^{\sigma, \rho}(\xi(\vartheta))= & \frac{1}{\Gamma(m-\sigma)} \int_{\mu}^{\vartheta} h^{m-\sigma-1}(\vartheta-\mu, \tau-\mu, \rho) \frac{D_{\mu, \omega}^{m, \rho}(\xi(\tau))}{\omega(\tau-\mu, \rho)} d \tau \\
& +\sum_{k=0}^{m-1} \frac{D_{\mu, \omega}^{m, \rho}(\xi(\mu))}{\Gamma(k-\sigma+1)} h^{k-\sigma}(\vartheta-\mu, 0, \rho),
\end{aligned}
$$

(ii) If $\xi \in W_{\omega, \nu}^{m, \rho}([\mu, \nu])$, then the RIFD of $\xi$ of order $\sigma$ exists and can be written in the form

$$
\begin{aligned}
\mathbb{D}_{\omega, \nu}^{\sigma, \rho}(\xi(\vartheta))= & \frac{(-1)^{m}}{\Gamma(m-\sigma)} \int_{\vartheta}^{v} h^{m-\sigma-1}(\nu-\vartheta, v-\tau, \rho) \frac{D_{\omega, \nu}^{m, \rho}(\xi(\tau))}{\omega(\nu-\tau, \rho)} d \tau \\
& +\sum_{k=0}^{m-1} \frac{(-1)^{k} D_{\omega, \nu}^{m, \rho}(\xi(\mu))}{\Gamma(k-\sigma+1)} h^{k-\sigma}(v-\vartheta, 0, \rho) .
\end{aligned}
$$

Proof We prove (i); (ii) can be proved similarly.

Let $\xi \in W_{\mu, \omega}^{m, \rho}([\mu, \nu])$. Then, by Lemma 6 , $\xi$ has the form

$$
\begin{aligned}
\xi(\vartheta)= & \frac{1}{\Gamma(m)} \int_{\mu}^{\vartheta} h^{m-1}(\vartheta-\mu, \tau-\mu, \rho) \frac{D_{\mu, \omega}^{k, \rho}(\xi(\tau))}{\omega(\tau-\mu, \rho)} d \tau \\
& +\sum_{k=1}^{m-1} \frac{D_{\mu, \omega}^{k, \rho}(\xi(\mu))}{k !} h^{k}(\vartheta-\mu, 0, \rho) .
\end{aligned}
$$

By Definition 6 and Lemma 4 we have

$$
\begin{aligned}
\mathbb{D}_{\omega, \nu}^{\sigma, \rho}(\xi(\vartheta))= & \frac{1}{\Gamma(m) \Gamma(m-\sigma)} \\
& \times D_{\omega, \nu}^{\sigma, \rho}\left(\int_{\mu}^{\vartheta} \int_{\mu}^{\tau_{1}} h^{m-\sigma-1}\left(\vartheta-\mu, \tau_{1}-\mu, \rho\right) h^{m-1}\left(\tau_{1}-\mu, \tau_{2}-\mu, \rho\right)\right. \\
& \left.\times\left(D_{\mu, \omega}^{k, \rho}\left(\xi\left(\tau_{2}\right)\right)\right) \frac{d \tau_{2}}{\omega\left(\tau_{2}-\mu, \rho\right)} \frac{d \tau_{1}}{\omega\left(\tau_{1}-\mu, \rho\right)}\right) \\
& +\sum_{k=0}^{m-1} \frac{D_{\mu, \omega}^{k, \rho}(\xi(\mu))}{\Gamma(k-\sigma+1)} h^{k-\sigma}(\vartheta-\mu, 0, \rho) .
\end{aligned}
$$

The variable transformation $\varrho=\frac{h\left(\tau_{1}-\mu, \tau_{2}-\mu, \rho\right)}{h\left(\vartheta-\mu, \tau_{2}-\mu, \rho\right)}$ yields

$$
\begin{aligned}
\mathbb{D}_{\omega, \nu}^{\sigma, \rho}(\xi(\vartheta))= & \frac{1}{\Gamma(2 m-\sigma)} D_{\omega, \nu}^{m, \rho}\left(\int_{\mu}^{\vartheta} h^{2 m-\sigma-1}(\vartheta-\mu, \tau-\mu, \rho) \frac{D_{\mu, \omega}^{k, \rho}\left(\xi\left(\tau_{2}\right)\right)}{\omega\left(\tau_{-} \mu, \rho\right)} d \tau\right) \\
& +\sum_{k=0}^{m-1} \frac{D_{\mu, \omega}^{k, \rho}(\xi(\mu))}{\Gamma(k-\sigma+1)} h^{k-\sigma}(\vartheta-\mu, 0, \rho) .
\end{aligned}
$$

Finally, applying the operator $D_{\omega, v}^{m, \rho}$ to the integral in the right side of Eq. (42) we acquire formula (38). 


\subsection{The inverse advantages on $W_{\mu, \omega}^{m, \rho}$ and $W_{\omega, \nu}^{m, \rho}$}

Here we give some inverse advantages with the same order for the fractional LIFD, RIFD, LIFI, and RIFI. This will be done for functions in $W_{\mu, \omega}^{m, \rho}$ and $W_{\omega, v}^{m, \rho}$.

Theorem 4 Let $\operatorname{Re}(\sigma)>0, \xi \in W_{\mu, \omega}^{m, \rho}$, and $\zeta \in W_{\omega, \nu}^{m, \rho}$. Then:

(i) $\mathbb{D}_{\mu, \omega}^{\sigma, \rho}\left(\mathbb{I}_{\mu, \omega}^{\sigma, \rho}(\xi(\vartheta))\right)=\xi(\vartheta)$,

(ii) $\mathbb{D}_{\omega, \nu}^{\sigma, \rho}\left(\mathbb{I}_{\omega, \nu}^{\sigma, \rho}(\zeta(\vartheta))\right)=\zeta(\vartheta)$.

Proof According to Definitions 5-6, the change of integration order, the transformation $\varrho=\frac{h\left(\tau_{1}-\mu, \tau_{2}-\mu, \rho\right)}{h\left(\vartheta-\mu, \tau_{2}-\mu, \rho\right)}$, and formula (8), we have

$$
\begin{aligned}
\mathbb{D}_{\mu, \omega}^{\sigma, \rho} & \left.\mathbb{I}_{\mu, \omega}^{\sigma, \rho}(\xi(\vartheta))\right) \\
= & \frac{1}{\Gamma(\sigma) \Gamma(m-\sigma)} D_{\mu, \omega}^{m, \rho}\left[\int_{\mu}^{\vartheta} \int_{\mu}^{\tau_{1}} h^{m-\sigma}\left(\vartheta-\mu, \tau_{1}-\mu, \rho\right) h^{\sigma-1}\left(\vartheta-\mu, \tau_{1}-\mu, \rho\right)\right. \\
& \left.\times \frac{\xi\left(\tau_{2}\right) d \tau_{2}}{\omega\left(\tau_{2}-\mu, \rho\right)} \frac{d \tau_{1}}{\omega\left(\tau_{1}-\mu, \rho\right)}\right] \\
= & \frac{1}{\Gamma(\sigma) \Gamma(m-\sigma)} D_{\mu, \omega}^{m, \rho}\left[\int_{\mu}^{\vartheta} \int_{\tau_{2}}^{\vartheta} h^{m-\sigma}\left(\vartheta-\mu, \tau_{1}-\mu, \rho\right) h^{\sigma-1}\left(\vartheta-\mu, \tau_{1}-\mu, \rho\right)\right. \\
& \left.\times \frac{d \tau_{1}}{\omega\left(\tau_{1}-\mu, \rho\right)} \frac{\xi\left(\tau_{2}\right) d \tau_{2}}{\omega\left(\tau_{2}-\mu, \rho\right)}\right] \\
= & \frac{1}{\Gamma(\sigma) \Gamma(m-\sigma)}\left(\int_{0}^{1}(1-\varrho)^{m-\sigma-1} \varrho^{\sigma-1} d \varrho\right) D_{\mu, \omega}^{m, \rho}\left[\int_{\mu}^{\vartheta} h^{m-1}\left(\vartheta-\mu, \tau_{1}, \rho\right)\right. \\
& \left.\times \frac{\xi(\tau) d \tau}{\omega(\tau-\mu, \rho)}\right] \\
= & \frac{1}{\Gamma(\sigma)} D_{\mu, \omega}^{m, \rho}\left[\int_{\mu}^{\vartheta} h^{m-1}\left(\vartheta-\mu, \tau_{1}, \rho\right) \frac{\xi(\tau) d \tau}{\omega(\tau-\mu, \rho)}\right] \\
= & D_{\mu, \omega}^{m, \rho}\left[\mathbf{I}_{\mu, \omega}^{m, \rho}(\xi(\vartheta))\right] \\
= & \xi(\vartheta) .
\end{aligned}
$$

This proves (i), and (ii) can be proved analogously.

Theorem 5 Let $\operatorname{Re}(\sigma)>0, m=-[-\operatorname{Re}(\sigma)], \xi, \zeta \in N(\mu), \mathbb{I}_{\mu, \omega}^{\sigma, \rho}(\xi(\vartheta)) \in W_{\mu, \omega}^{m, \rho}$, and $\mathbb{I}_{\omega, \nu}^{\sigma, \rho}(\zeta(\vartheta)) \in W_{\mu, \omega}^{m, \rho}$. Then

$$
\mathbb{I}_{\mu, \omega}^{\sigma, \rho}\left(\mathbb{D}_{\mu, \omega}^{\sigma, \rho}(\xi(\vartheta))\right)=\xi(\vartheta)-\sum_{k=1}^{m} \frac{\mathbb{D}_{\mu, \omega}^{\sigma-k, \rho}(\xi(\mu))}{\Gamma(\sigma-k+1)} h^{\sigma-k}(\vartheta-\mu, 0, \rho),
$$

and

$$
\mathbb{I}_{\omega, \nu}^{\sigma, \rho}\left(\mathbb{D}_{\omega, \nu}^{\sigma, \rho}(\xi(\vartheta))\right)=\xi(\vartheta)-\sum_{k=1}^{m} \frac{(-1)^{\sigma-k} \mathbb{D}_{\omega, \nu}^{\sigma-k, \rho}(\xi(v))}{\Gamma(\sigma-k+1)} h^{\sigma-k}(\nu-\vartheta, 0, \rho) .
$$


Proof Let us prove formula (44). The proof of formula (45) can be obtained similarly. We have

$$
\begin{aligned}
\mathbb{I}_{\mu, \omega}^{\sigma, \rho}\left(\mathbb{D}_{\mu, \omega}^{\sigma, \rho}(\xi(\vartheta))\right)= & \frac{1}{\Gamma(\sigma)} \int_{\mu}^{\vartheta} h^{\sigma-1}(\vartheta-\mu, \tau-\mu, \rho)\left(D_{\mu, \omega}^{m, \rho}\left(\mathbb{I}_{\mu, \omega}^{m-\sigma, \rho}(\xi(\tau))\right) \frac{d \tau}{\omega(\tau-\mu, \rho)}\right. \\
= & \frac{D_{\mu, \omega}^{1, \rho}}{\Gamma(\sigma+1)} \int_{\mu}^{\vartheta} h^{\sigma}(\vartheta-\mu, \tau-\mu, \rho)\left(D_{\mu, \omega}^{m, \rho}\left(\mathbb{I}_{\mu, \omega}^{m-\sigma, \rho}(\xi(\tau))\right) \frac{d \tau}{\omega(\tau-\mu, \rho)}\right. \\
= & D_{\mu, \omega}^{1, \rho}\left[\frac{1}{\Gamma(\sigma-m+1)} \int_{\mu}^{\vartheta} h^{\sigma-n}(\vartheta-\mu, \tau-\mu, \rho)\left(\mathbb{I}_{\mu, \omega}^{m-\sigma, \rho}(\xi(\tau))\right)\right. \\
& \left.-\sum_{k=1}^{m} \frac{D_{\mu, \omega}^{m-k, \rho}\left(\mathbb{I}_{\mu, \omega}^{m-\sigma, \rho}(\xi(\mu))\right)}{\Gamma(\sigma-k+2)} h^{\sigma-k+1}(\vartheta-\mu, 0, \rho)\right] \\
= & D_{\mu, \omega}^{1, \rho}\left[\mathbb{I}_{\mu, \omega}^{\sigma-m+1, \rho}\left(\mathbb{I}_{\mu, \omega}^{m-\sigma, \rho}(\xi(\vartheta))\right)-\sum_{k=1}^{m} \frac{D_{\mu, \omega}^{m-k, \rho}\left(\mathbb{I}_{\mu, \omega}^{m-\sigma, \rho}(\xi(\mu))\right)}{\Gamma(\sigma-k+2)}\right. \\
& \left.\times h^{\sigma-k+1}(\vartheta-\mu, 0, \rho)\right] \\
= & D_{\mu, \omega}^{1, \rho}\left[\mathbb{I}_{\mu, \omega}^{1, \rho}(\xi(\vartheta))-\sum_{k=1}^{m} \frac{D_{\mu, \omega}^{m-k, \rho}\left(\mathbb{I}_{\mu, \omega}^{m-\sigma, \rho}(\xi(\mu))\right)}{\Gamma(\sigma-k+2)} h^{\sigma-k+1}(\vartheta-\mu, 0, \rho)\right] \\
= & \xi(\vartheta)-\sum_{k=1}^{m} \frac{\mathbb{D}_{\mu, \omega}^{\sigma-k, \rho}(\xi(\mu))}{\Gamma(\sigma-k+1)} h^{\sigma-k}(\vartheta-\mu, 0, \rho) .
\end{aligned}
$$

Here we have employed the integration by parts $m$ times and Lemma 2 .

\section{Improved fractional derivatives in the Caputo sense}

Here we define the improved fractional derivatives in the Caputo sense. These types of derivatives are new and generalize many derivatives proposed in the literature. Moreover, we discuss the advantages of these derivatives via the results obtained in Sect. 3.

Definition 9 Let $\rho>0, \operatorname{Re}(\sigma) \geq 0$, and $m=[\operatorname{Re}(\sigma)]+1$. If $\xi \in W_{\mu, \omega}^{m, \rho}([\mu, \nu])$ and $\zeta \in$ $W_{\omega, \nu}^{m, \rho}([\mu, \nu])$, then we define the Caputo LIFD and RIFD, respectively, as

$$
C_{\mathbb{D}_{\mu, \omega}^{\sigma, \rho}}(\xi(\vartheta))=\mathbb{D}_{\mu, \omega}^{\sigma, \rho}\left(\xi(\vartheta)-\sum_{k=0}^{m-1} \frac{D_{\mu, \omega}^{k, \rho}(\xi(\mu))}{k !} h^{k}(\vartheta-\mu, 0, \rho)\right)
$$

and

$$
C_{\mathbb{D}_{\omega, \nu}^{\sigma, \rho}}(\zeta(\vartheta))=\mathbb{D}_{\omega, \nu}^{\sigma, \rho}\left(\zeta(\vartheta)-\sum_{k=0}^{m-1} \frac{(-1)^{k} D_{\omega, \nu}^{k, \rho}(\zeta(\nu))}{k !} h^{k}(v-\vartheta, 0, \rho)\right) .
$$

The following theorem gives alternative definitions of the Caputo LIFD and RIFD via the LIFI and RIFI. 
Theorem 6 Let $\rho>0, \operatorname{Re}(\sigma) \geq 0$, and $m=[\operatorname{Re}(\sigma)]+1$. If $\xi \in W_{\mu, \omega}^{m, \rho}([\mu, \nu])$ and $\zeta \in$ $W_{\omega, v}^{m, \rho}([\mu, \nu])$, then the Caputo LIFD and RIFD can be redefined, respectively, as

$$
\begin{aligned}
C_{\mathbb{D}_{\mu, \omega}^{\sigma, \rho}(\xi(\vartheta))} & =\frac{1}{\Gamma(m-\sigma)} \int_{\mu}^{\vartheta} h^{m-\sigma-1}(\vartheta-\mu, \tau-\mu, \rho) \frac{D_{\mu, \omega}^{m, \rho}(\xi(\tau)) d \tau}{\omega(\tau-\mu, \rho)} \\
& =\mathbb{I}_{\mu, \omega}^{m-\sigma, \rho}\left(D_{\mu, \omega}^{m, \rho}(\xi(\vartheta))\right),
\end{aligned}
$$

and

$$
\begin{aligned}
C_{\mathbb{D}_{\omega, \nu}^{\sigma, \rho}}(\zeta(\vartheta)) & =\frac{(-1)^{m}}{\Gamma(m-\sigma)} \int_{\vartheta}^{v} h^{m-\sigma-1}(v-\vartheta, v-\tau, \rho) \frac{D_{\omega, \nu}^{m, \rho}(\xi(\tau)) d \tau}{\omega(v-\tau, \rho)} \\
& =\mathbb{I}_{\omega, v}^{m-\sigma, \rho}\left((-1)^{m} D_{\omega, \nu}^{m, \rho}(\xi(\vartheta))\right) .
\end{aligned}
$$

Proof By (47), Lemma 4, and Theorem 3 we have

$$
\begin{aligned}
C_{\mathbb{D}_{\mu, \omega}^{\sigma, \rho}(\xi(\vartheta))} & =\mathbb{D}_{\mu, \omega}^{\sigma, \rho}(\xi(\vartheta))-\sum_{k=0}^{m-1} \frac{D_{\mu, \omega}^{k, \rho}(\xi(\mu))}{k !} \frac{\Gamma(k+1)}{\Gamma(k-\sigma+1)} h^{k-\sigma}(\vartheta-\mu, 0, \rho) \\
& =\mathbb{D}_{\mu, \omega}^{\sigma, \rho}(\xi(\vartheta))-\sum_{k=0}^{m-1} \frac{D_{\mu, \omega}^{k, \rho}(\xi(\mu))}{\Gamma(k-\sigma+1)} h^{k-\sigma}(\vartheta-\mu, 0, \rho) \\
& =\frac{1}{\Gamma(m-\sigma)} \int_{\mu}^{\vartheta} h^{m-\sigma-1}(\vartheta-\mu, \tau-\mu, \rho) \frac{D_{\mu, \omega}^{m, \rho}(\xi(\tau)) d \tau}{\omega(\tau-\mu, \rho)} \\
& =\mathbb{I}_{\mu, \omega}^{m-\sigma, \rho}\left(D_{\mu, \omega}^{m, \rho}(\xi(\vartheta))\right) .
\end{aligned}
$$

Formula (49) can be analogously proved by employing (48), Lemma 4, and Theorem 3 .

Remark 7 The Caputo LIFD and RIFD generalize some fractional differential operators proposed in the literature. The following cases show this fact.

(1) If $\omega(\vartheta, \rho)=\vartheta^{1-\rho}$, then $h(\vartheta, 0, \rho)=\frac{\vartheta^{\rho}}{\rho}, D_{\mu, \omega}^{\rho}(\xi(\vartheta))=(\vartheta-\mu)^{1-\rho} \frac{d \xi}{d \vartheta}$, and

$D_{\omega, v}^{\rho}(\xi(\vartheta))=(v-\vartheta)^{1-\rho} \frac{d \xi}{d \vartheta}$. Hence, the Caputo LIFD and RIFD in (47) and (48)

coincide with the left and right Caputo fractional derivatives defined by Jarad et al.

[31], respectively.

(2) If $\omega(\vartheta, \rho)=\vartheta^{1-\rho}$ and $\mu=v=0$, then the Caputo LIFD and RIFD in (47) and (48)

coincide with the left and right Caputo modifications of fractional derivatives defined by Jarad et al. [30].

(3) If $\omega(\vartheta, \rho)=\vartheta^{1-\rho}, \rho=1$, and $\mu=v=0$, then $h(\vartheta, 0, \rho)=\vartheta$, and $D_{\mu, \omega}^{\rho}(\xi(\vartheta))=D_{\omega, \nu}^{\rho}(\xi(\vartheta))=\frac{d \xi}{d \vartheta}$. Hence, respectively, the Caputo LIFD and RIFD in (47) and (48) coincide with the left and right Caputo fractional derivatives defined in $[2,44]$.

(4) If $\omega(\vartheta, \rho)=\vartheta^{1-\rho}, \mu=v=0$, and $\rho \rightarrow 0$, then $h(\vartheta, 0, \rho) \rightarrow \ln (\vartheta), D_{\mu, \omega}^{\rho}(\xi(\vartheta)) \rightarrow \vartheta \frac{d \xi}{d \vartheta}$, and $D_{\omega, v}^{\rho}(\xi(\vartheta)) \rightarrow-\vartheta \frac{d \xi}{d \vartheta}$. Hence, respectively, the Caputo LIFD and RIFD in (47) and (48) coincide with the fractional left and right derivatives of Caputo-Hadamard defined in [12].

The following two lemmas are beneficial tools for showing the inverse and composition advantages of the Caputo LIFD and RIFD. 
Lemma 8 Let $0<\operatorname{Re}(\sigma) \notin \mathbb{N}, m=[\operatorname{Re}(\sigma)]+1$, and $\xi \in C[\mu, \nu]$. Then $\mathbb{I}_{\mu, \omega}^{\sigma-k, \rho}(\xi(\mu))=0$ and $\mathbb{I}_{\omega, v}^{\sigma-k, \rho}(\xi(\nu))=0$ for $k \in\{0,1, \ldots, m-1\}$.

Proof Using Definition 5, we have

$$
\left|\mathbb{I}_{\mu, \omega}^{\sigma-k, \rho}(\xi(\vartheta))\right| \leq \frac{\|\xi\|_{C}}{|\Gamma(\sigma-k)|(\operatorname{Re}(\sigma)-k)} h^{\operatorname{Re}(\sigma)-k}(\vartheta-\mu, 0, \rho) .
$$

Replacing $\vartheta$ by $\mu$ gives $\mathbb{I}_{\mu, \omega}^{\sigma-k, \rho}(\xi(\mu))=0$. The second equality can be shown similarly.

Lemma 9 Let $\operatorname{Re}(\sigma) \geq 0, m=[\operatorname{Re}(\sigma)]+1$, and $D_{\mu, \omega}^{m, \rho}, D_{\omega, \nu}^{m, \rho} \in C[\mu, \nu]$. Then ${ }^{C} \mathbb{D}_{\mu, \omega}^{\sigma, \rho}(\xi(\mu))=0$ and ${ }^{C} \mathbb{D}_{\omega, \nu}^{\sigma, \rho}(\xi(\nu))=0$.

Proof From Theorem 6 we get

$$
\left|{ }^{C} \mathbb{D}_{\mu, \omega}^{\sigma, \rho}(\xi(\vartheta))\right| \leq \frac{\left\|D_{\mu, \omega}^{m, \rho}\right\|_{C}}{|\Gamma(m-\sigma)|(m-\operatorname{Re}(\sigma))} h^{m-\operatorname{Re}(\sigma)}(\vartheta-\mu, 0, \rho)
$$

and

$$
\left|{ }^{C} \mathbb{D}_{\omega, \nu}^{\sigma, \rho}(\xi(\vartheta))\right| \leq \frac{\left\|D_{\omega, \nu}^{m, \rho}\right\|_{C}}{|\Gamma(m-\sigma)|(m-\operatorname{Re}(\sigma))} h^{m-\operatorname{Re}(\sigma)}(v-\vartheta, 0, \rho) .
$$

Hence the results can be acquired by replacing $\vartheta$ by $\mu$ and $\nu$, respectively.

Now we give the inverse advantages of the Caputo LIFD and RIFD.

Theorem 7 Suppose $\operatorname{Re}(\sigma)>0, m=[\operatorname{Re}(\sigma)]+1$, and $\xi \in C[\mu, \nu]$.

(i) If $\operatorname{Re}(\sigma) \notin \mathbb{N}$ or $\sigma \in \mathbb{N}$, then

$$
{ }^{C} \mathbb{D}_{\mu, \omega}^{\sigma, \rho}\left(\mathbb{I}_{\mu, \omega}^{\sigma, \rho}(\xi(\vartheta))\right)=\xi(\vartheta) \quad \text { and } \quad{ }^{C} \mathbb{D}_{\omega, \nu}^{\sigma, \rho}\left(\mathbb{I}_{\omega, \nu}^{\sigma, \rho}(\xi(\vartheta))\right)=\xi(\vartheta)
$$

(ii) If $\operatorname{Re}(\sigma) \in \mathbb{N}$, then

$$
C^{C} \mathbb{D}_{\mu, \omega}^{\sigma, \rho}\left(\mathbb{I}_{\mu, \omega}^{\sigma, \rho}(\xi(\vartheta))\right)=\xi(\vartheta)-\frac{\mathbb{I}_{\mu, \omega}^{\sigma+1-m, \rho}(\xi(\mu))}{\Gamma(m-\sigma)} h^{m-\sigma}(\vartheta-\mu, 0, \rho),
$$

and

$$
{ }^{C} \mathbb{D}_{\omega, \nu}^{\sigma, \rho}\left(\mathbb{I}_{\omega, \nu}^{\sigma, \rho}(\xi(\vartheta))\right)=\xi(\vartheta)-\frac{\mathbb{I}_{\omega, \nu}^{\sigma+1-m, \rho}(\xi(\nu))}{\Gamma(m-\sigma)} h^{m-\sigma}(\nu-\vartheta, 0, \rho) .
$$

Proof According to Definition 9, Lemmas 4-5, and Theorem 4, we have

$$
\begin{aligned}
C_{\mathbb{D}_{\mu, \omega}^{\sigma, \rho}}^{\sigma}\left(\mathbb{I}_{\mu, \omega}^{\sigma, \rho}(\xi(\vartheta))\right) & =\mathbb{D}_{\mu, \omega}^{\sigma, \rho}\left(\mathbb{I}_{\mu, \omega}^{\sigma, \rho}(\xi(\vartheta))\right)-\sum_{k=0}^{m-1} \frac{D_{\mu, \omega}^{k, \rho}\left(\mathbb{I}_{\mu, \omega}^{\sigma, \rho}(\xi(\mu))\right)}{\Gamma(k-\sigma+1)} h^{k-\sigma}(\vartheta-\mu, 0, \rho) \\
& =\xi(\vartheta)-\sum_{k=0}^{m-1} \frac{\mathbb{I}_{\mu, \omega}^{\sigma, \rho}(\xi(\mu))}{\Gamma(k-\sigma+1)} h^{k-\sigma}(\vartheta-\mu, 0, \rho) .
\end{aligned}
$$

Now the proof can be completed as follows. 
(i) Let $\operatorname{Re}(\sigma) \notin \mathbb{N}$. Then by Lemma 8 we have $\mathbb{I}_{\mu, \omega}^{\sigma, \rho}(\xi(\mu))=0$ for $k \in\{0,1, \ldots, m-1\}$. Hence, the first equality in (55) is proved. The second equality can be proved by the same procedure. The case of $\sigma \in \mathbb{N}$ is trivial, because we return to the integer-order case.

(ii) Let $\operatorname{Re}(\sigma) \in \mathbb{N}$, then it is easy to repeat the steps of Lemma 8 and conclude that $\mathbb{I}_{\mu, \omega}^{\sigma, \rho}(\xi(\mu))=0$ for $k \in\{0,1, \ldots, m-1\}$. Thus (56) is proved, and (57) can be proved similarly.

Theorem 8 Suppose $\operatorname{Re}(\sigma)>0, m=[\operatorname{Re}(\sigma)]+1, \xi \in W_{\mu, \omega}^{m, \rho}$, and $\zeta \in W_{\omega, \nu}^{m, \rho}$. Then

$$
\mathbb{I}_{\mu, \omega}^{\sigma, \rho}\left({ }^{C} \mathbb{D}_{\mu, \omega}^{\sigma, \rho}(\xi(\vartheta))\right)=\xi(\vartheta)-\sum_{k=0}^{m-1} \frac{D_{\mu, \omega}^{k, \rho}(\xi(\mu))}{k !} h^{k}(\vartheta-\mu, 0, \rho)
$$

and

$$
\mathbb{I}_{\omega, \mu}^{\sigma, \rho}\left({ }^{C} \mathbb{D}_{\omega, \nu}^{\sigma, \rho}(\zeta(\vartheta))\right)=\zeta(\vartheta)-\sum_{k=0}^{m-1} \frac{(-1)^{k} D_{\omega, \nu}^{k, \rho}(\zeta(\nu))}{k !} h^{k}(v-\vartheta, 0, \rho)
$$

Proof We prove formula (59), and formula (60) can be proved likewise. According to Theorem 6 and Lemma 2, we have

$$
\begin{aligned}
\mathbb{I}_{\mu, \omega}^{\sigma, \rho}\left({ }^{C} \mathbb{D}_{\mu, \omega}^{\sigma, \rho}(\xi(\vartheta))\right) & =\mathbb{I}_{\mu, \omega}^{\sigma, \rho}\left(\mathbb{I}_{\mu, \omega}^{m-\sigma, \rho}\left(D_{\mu, \omega}^{m, \rho}(\xi(\vartheta))\right)\right)=\mathbb{I}_{\mu, \omega}^{m, \rho}\left(D_{\mu, \omega}^{m, \rho}(\xi(\vartheta))\right) \\
& =\frac{1}{\Gamma(m)} \int_{\mu}^{\vartheta} h^{m-1}(\vartheta-\mu, \tau-\mu, \rho) \frac{D_{\mu, \omega}^{m, \rho}(\xi(\tau))}{\omega(\tau-\mu, \rho)} d \tau \\
& =\frac{1}{\Gamma(m+1)} D_{\mu, \omega}^{1, \rho}\left[\int_{\mu}^{\vartheta} h^{m}(\vartheta-\mu, \tau-\mu, \rho) \frac{D_{\mu, \omega}^{m, \rho}(\xi(\tau))}{\omega(\tau-\mu, \rho)} d \tau\right]
\end{aligned}
$$

Using the integration by parts $m$ times yields

$$
\begin{aligned}
\mathbb{I}_{\mu, \omega}^{\sigma, \rho}\left({ }^{C} \mathbb{D}_{\mu, \omega}^{\sigma, \rho}(\xi(\vartheta))\right) & =D_{\mu, \omega}^{1, \rho}\left[\mathbf{I}_{\mu, \omega}^{1, \rho}(\xi(\vartheta))-\sum_{i=1}^{m} \frac{D_{\mu, \omega}^{m-i, \rho}(\xi(\mu))}{\Gamma(m-i+2)} h^{m-i+1}(\vartheta-\mu, 0, \rho)\right] \\
& =\xi(\vartheta)-\sum_{i=1}^{m} \frac{D_{\mu, \omega}^{m-i, \rho}(\xi(\mu))}{\Gamma(m-i+1)} h^{m-i}(\vartheta-\mu, 0, \rho) \\
& =\xi(\vartheta)-\sum_{k=0}^{m-1} \frac{D_{\mu, \omega}^{k, \rho}(\xi(\mu))}{k !} h^{k}(\vartheta-\mu, 0, \rho) .
\end{aligned}
$$

This proves formula (59)

The following theorem gives the composite of two Caputo LIFDs and RIFDs.

Theorem 9 Let $\xi \in W_{\mu, \omega}^{m_{1}+m_{2}}([\mu, v]), \zeta \in W_{\omega, v}^{m_{1}+m_{2}}([\mu, \nu]), \operatorname{Re}\left(\sigma_{1}\right) \geq 0, \operatorname{Re}\left(\sigma_{2}\right) \geq 0, \operatorname{Re}\left(\sigma_{1}\right) \in$ $\left(m_{1}-1, m_{1}\right]$, and $\operatorname{Re}\left(\sigma_{2}\right) \in\left(m_{2}-1, m_{2}\right]$. Then:

(i) ${ }^{C} \mathbb{D}_{\mu, \omega}^{\sigma_{1}, \rho}\left({ }^{C} \mathbb{D}_{\mu, \omega}^{\sigma_{2}, \rho}(\xi(\vartheta))\right)={ }^{C} \mathbb{D}_{\mu, \omega}^{\sigma_{1}+\sigma_{2}, \rho}(\xi(\vartheta))$,

(ii) ${ }^{C} \mathbb{D}_{\omega, v}^{\sigma_{1}, \rho}\left({ }^{C} \mathbb{D}_{\omega, v}^{\sigma_{2}, \rho}(\zeta(\vartheta))\right)={ }^{C} \mathbb{D}_{\omega, v}^{\sigma_{1}+\sigma_{2}, \rho}(\zeta(\vartheta))$. 
Proof Using Theorem 6, Lemma 5, and Lemma 2, we have

$$
\begin{aligned}
{ }^{C} \mathbb{D}_{\mu, \omega}^{\sigma_{1}, \rho}\left({ }^{C} \mathbb{D}_{\mu, \omega}^{\sigma_{2}, \rho}(\xi(\vartheta))\right) & ={ }^{C} \mathbb{D}_{\mu, \omega}^{\sigma_{1}, \rho}\left(\mathbb{I}_{\mu, \omega}^{m_{2}-\sigma_{2}, \rho}\left(D_{\mu, \omega}^{m_{2}, \rho}(\xi(\vartheta))\right)\right) \\
& =\mathbb{I}_{\mu, \omega}^{m_{1}-\sigma_{1}, \rho}\left(D_{\mu, \omega}^{m_{1}, \rho}\left(\mathbb{I}_{\mu, \omega}^{m_{2}-\sigma_{2}, \rho}\left(D_{\mu, \omega}^{m_{2}, \rho}(\xi(\vartheta))\right)\right)\right) \\
& =\mathbb{I}_{\mu, \omega}^{m_{2}-\left(\sigma_{1}+\sigma_{2}\right), \rho}\left(D_{\mu, \omega}^{m_{2}, \rho}(\xi(\vartheta))\right) \\
& ={ }^{C} \mathbb{D}_{\mu, \omega}^{\sigma_{1}+\sigma_{2}, \rho}(\xi(\vartheta)) .
\end{aligned}
$$

This proves (i), and (ii) can be proved likewise.

\section{Applications to electrical circuits of fractional orders}

In this part, we provide some application examples to justify the importance and merit of the novel IF operators. In particular, we discuss the resistor-capacitor ( $\mathfrak{R} \mathfrak{C})$ electrical circuits in the framework of the Caputo LIFD and the LIFI. New analytical solutions that describe the voltage are gained in the cases of constant and periodic sources. Also, some 2D numerical simulations are displayed to illustrate the impact of the Caputo LIFD and the LIFI. Finally, we can see that the obtained results include some previously acquired results as particular cases.

Example 1 (The fractional $\mathfrak{R C}$ electrical circuit with constant source) Consider a fractional $\mathfrak{R C}$ electrical circuit described by

$$
\left\{\begin{array}{l}
{ }^{C} \mathbb{D}_{0, \omega}^{\sigma, \rho}(\mathfrak{V}(\vartheta))+\Phi_{\sigma, \rho} \mathfrak{V}(\vartheta)=\Psi_{\sigma, \rho}, \quad \vartheta \in(0, \infty), \\
\mathfrak{V}(0)=\mathfrak{V}_{0},
\end{array}\right.
$$

where $\sigma, \rho \in(0,1],{ }^{C} \mathbb{D}_{\mu, \omega}^{\sigma, \rho}$ is the Caputo LIFD, $\mathfrak{V}$ is the voltage, $\mathfrak{V}_{0}$ is the initial voltage, $\Phi_{\sigma, \rho}=1 /(\mathfrak{R C})^{\sigma \rho}, \Psi_{\sigma, \rho}=\mathfrak{E}_{0} /(\mathfrak{R C})^{\sigma \rho}, \mathfrak{E}_{0}$ is a constant source, and $\mathfrak{R}^{\sigma \rho}$ and $\mathfrak{C}^{\sigma \rho}$ are nonlocal parameters, the resistance and capacitance, respectively. The reason for using the nonlocal parameters $\mathfrak{R}^{\sigma \rho}$ and $\mathfrak{C}^{\sigma \rho}$ instead of the traditional parameters is retaining the fractional dimensionality of the equation.

Applying the LIFI operator to (64) and using Theorem 8 and Lemma 3, we have

$$
\mathfrak{V}(\vartheta)=\mathfrak{V}_{0}+\frac{\Psi_{\sigma, \rho}}{\Gamma(\sigma+1)} h^{\sigma}(\vartheta, 0, \rho)-\Phi_{\sigma, \rho} \mathbb{I}_{0, \omega}^{\sigma, \rho}(\mathfrak{V}(\vartheta)) .
$$

Hence

$$
\mathfrak{V}_{m+1}(\vartheta)=\mathfrak{V}_{0}+\frac{\Psi_{\sigma, \rho}}{\Gamma(\sigma+1)} h^{\sigma}(\vartheta, 0, \rho)-\Phi_{\sigma, \rho} \mathbb{I}_{0, \omega}^{\sigma, \rho}\left(\mathfrak{V}_{m}(\vartheta)\right), \quad m=0,1,2, \ldots
$$

For $m=0$, we have

$$
\mathfrak{V}_{1}(\vartheta)=\mathfrak{V}_{0}+\frac{\Psi_{\sigma, \rho}}{\Gamma(\sigma+1)} h^{\sigma}(\vartheta, 0, \rho)-\Phi_{\sigma, \rho} \mathbb{I}_{0, \omega}^{\sigma, \rho}\left(\mathfrak{V}_{0}(\vartheta)\right) .
$$

According to Lemma 3, we get

$$
\mathfrak{V}_{1}(\vartheta)=\mathfrak{V}_{0}\left(1-\frac{\Phi_{\sigma, \rho}}{\Gamma(\sigma+1)} h^{\sigma}(\vartheta, 0, \rho)\right)+\frac{\Psi_{\sigma, \rho}}{\Gamma(\sigma+1)} h^{\sigma}(\vartheta, 0, \rho) .
$$


For $m=2$, we have

$$
\mathfrak{V}_{2}(\vartheta)=\mathfrak{V}_{0}+\frac{\Psi_{\sigma, \rho}}{\Gamma(\sigma+1)} h^{\sigma}(\vartheta, 0, \rho)-\Phi_{\sigma, \rho} \mathbb{I}_{0, \omega}^{\sigma, \rho}\left(\mathfrak{V}_{1}(\vartheta)\right)
$$

Inserting Eq. (68) into (69) and using Lemma 3 yield

$$
\begin{aligned}
\mathfrak{V}_{1}(\vartheta)= & \mathfrak{V}_{0}\left(1-\frac{\Phi_{\sigma, \rho}}{\Gamma(\sigma+1)} h^{\sigma}(\vartheta, 0, \rho)+\frac{\Phi_{\sigma, \rho}^{2}}{\Gamma(2 \sigma+1)} h^{2 \sigma}(\vartheta, 0, \rho)\right) \\
& +\Psi_{\sigma, \rho} h^{\sigma}(\vartheta, 0, \rho)\left(\frac{1}{\Gamma(\sigma+1)}+\frac{\Phi_{\sigma, \rho}}{\Gamma(2 \sigma+1)} h^{\sigma}(\vartheta, 0, \rho)\right) .
\end{aligned}
$$

Repeating the same steps $m-2$ times, we get

$$
\begin{aligned}
\mathfrak{V}_{m}(\vartheta)= & \mathfrak{V}_{0} E_{\sigma, 1}\left(-\Phi_{\sigma, \rho} h^{\sigma}(\vartheta, 0, \rho)\right) \\
& +\Psi_{\sigma, \rho} h^{\sigma}(\vartheta, 0, \rho) \sum_{k=0}^{n} \frac{(-1)^{k} \Phi_{\sigma, \rho}^{k}}{(k+1) \sigma \Gamma(k \sigma+\sigma)} h^{k \sigma}(\vartheta, 0, \rho) .
\end{aligned}
$$

So, as $m \rightarrow \infty$, we have the solution of (64) of the form

$$
\begin{aligned}
\mathfrak{V}(\vartheta)= & \mathfrak{V}_{0} E_{\sigma, 1}\left(-\Phi_{\sigma, \rho} h^{\sigma}(\vartheta, 0, \rho)\right) \\
& +\Psi_{\sigma, \rho} h^{\sigma}(\vartheta, 0, \rho) \sum_{k=0}^{\infty} \frac{(-1)^{k} \Phi_{\sigma, \rho}^{k}}{(k+1) \sigma \Gamma(k \sigma+\sigma)} h^{k \sigma}(\vartheta, 0, \rho),
\end{aligned}
$$

where $E_{\sigma, \rho}$ is the biparameterized Mittag-Leffler function. The Appendix provides the definition and some required properties of the function $E_{\sigma, \rho}$.

Now, for $\mathfrak{R}=1 \mathrm{Ohm}, C=20$ Farad, $\mathfrak{E}_{0}=10$ Watts, and $\omega(\vartheta, \rho)=e^{(1-\rho) \vartheta}$, the behavior of the voltage $\mathfrak{V}(\vartheta)$ in (72) is exhibited in Fig. 1 for various values of the orders $\sigma$ and $\rho$. Also, Fig. 1 shows the gross effect of the Caputo $\operatorname{LIFD}^{C} \mathbb{D}_{0, \omega}^{\sigma, \rho}$ on the voltage behavior of the fractional $\mathfrak{R C}$ electrical circuit (64), whereas the fractional order $\sigma$ describes irrevocable dispersion effects like internal or ohmic friction, and the conformable order $\rho$ is responsible for speeding up or slowing down the decrease in voltage.

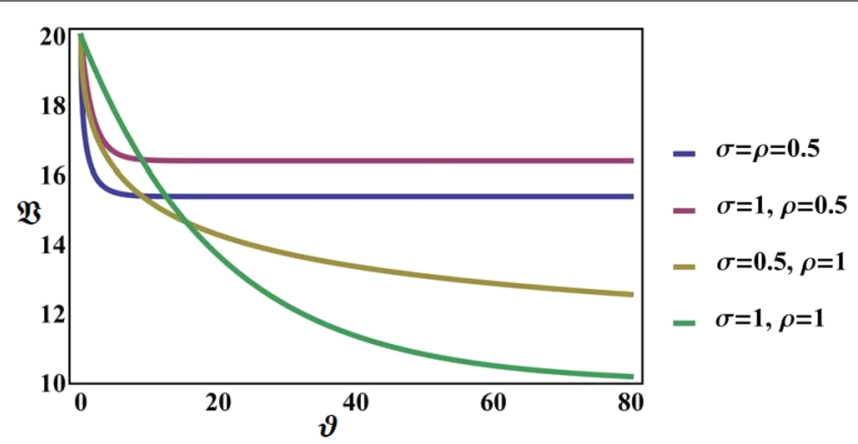

Figure 1 The behavior of the voltage $\mathfrak{V}(\vartheta)$ in Eq. (72) for some picked values of $\sigma$ and $\rho$ 


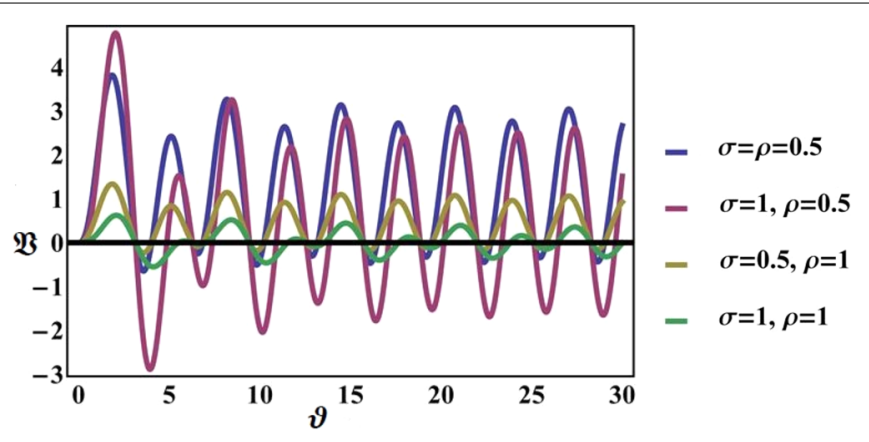

Figure 2 Simulation of the voltage $\mathfrak{V}(\vartheta)$ in Eq. (76) for some picked values of $\sigma$ and $\rho$

Example 2 (The fractional $\mathfrak{R C}$ electrical circuit with periodic source) Consider the fractional $\mathfrak{R C}$ electrical circuit given by

$$
\left\{\begin{array}{l}
{ }^{C} \mathbb{D}_{0, \omega}^{\sigma, \rho}(\mathfrak{V}(\vartheta))+\Phi_{\sigma, \rho} \mathfrak{V}(\vartheta)=\Psi_{\sigma, \rho} \sin (\varepsilon \vartheta), \quad \vartheta \in(0, \infty) \\
\mathfrak{V}(0)=\mathfrak{V}_{0}
\end{array}\right.
$$

where $\Phi_{\sigma, \rho}$ and $\Psi_{\sigma, \rho}$ are those defined in Example 1, and $\varepsilon \in \mathbb{R}$.

Taking the Laplace transform of Eq. (73), we get

$$
\begin{aligned}
& \mathfrak{D}_{\sigma, \rho}\left(\rho^{\sigma} h^{\sigma}(\varpi, 0, \rho) \mathfrak{V}(\varpi)-\rho^{\sigma-\frac{1}{\rho}} h^{\sigma-\frac{1}{\rho}}(\vartheta, 0, \rho) \mathfrak{V}(0)\right) \\
& \quad=\Psi_{\sigma, \rho} \frac{\varepsilon}{\varpi^{2}+\varepsilon^{2}}-\Phi_{\sigma, \rho} \mathfrak{V}(\varpi)
\end{aligned}
$$

where $\mathfrak{D}_{\sigma, \rho}=\frac{\rho^{\sigma} \Gamma(\sigma \rho)}{\Gamma(\rho)}$. Simplifying Eq. (74) and using the initial data give

$$
\mathfrak{V}(\varpi)=\mathfrak{V}_{0} \frac{\rho^{\sigma-\frac{1}{\rho}} h^{\sigma-\frac{1}{\rho}}(\vartheta, 0, \rho)}{\rho^{\sigma} h^{\sigma}(\vartheta, 0, \rho)+\frac{\Phi_{\sigma, \rho}}{\mathfrak{D}_{\sigma, \rho}}}+\frac{\varepsilon \Psi_{\sigma, \rho}}{\mathfrak{D}_{\sigma, \rho}\left(\varpi^{2}+\varepsilon^{2}\right)\left(\rho^{\sigma} h^{\sigma}(\vartheta, 0, \rho)+\frac{\Phi_{\sigma, \rho}}{\mathfrak{D}_{\sigma, \rho}}\right)} .
$$

Hence by taking the inverse Laplace transform we have

$$
\begin{aligned}
\mathfrak{V}(\vartheta)= & \mathfrak{V}_{0} \vartheta^{\frac{1}{\rho}-1} E_{\sigma, \frac{1}{\rho}}\left(-\frac{\Phi_{\sigma, \rho}}{\mathfrak{D}_{\sigma, \rho}} \rho^{\sigma} h^{\sigma}(\vartheta, 0, \rho)\right) \\
& +\frac{\Psi_{\sigma, \rho}}{\mathfrak{D}_{\sigma, \rho}} \int_{0}^{\vartheta} \rho^{\sigma-1} h^{\sigma-1}(\tau, 0, \rho) E_{\sigma, \sigma}\left(-\frac{\Phi_{\sigma, \rho}}{\mathfrak{D}_{\sigma, \rho}} \rho^{\sigma} h^{\sigma}(\tau, 0, \rho)\right) \sin (\varepsilon(\vartheta-\tau)) d \tau .
\end{aligned}
$$

For $\mathfrak{R}=1$ Ohm, $\mathfrak{C}=20$ Farad, $\mathfrak{E}_{0}=10$ Watts, $\varepsilon=1$, and $\omega(\vartheta, \rho)=\frac{1}{2}\left(1+\vartheta^{\rho(1-\rho)}\right)$, the behavior of the voltage $\mathfrak{V}(\vartheta)$ in (76) is shown in Fig. 2 for various values of the orders $\sigma$ and $\rho$. Further, Fig. 1 clarifies the overall effect of the Caputo LIFD ${ }^{C} \mathbb{D}_{0, \omega}^{\sigma, \rho}$ on the voltage behavior of the fractional $\mathfrak{R C}$ electrical circuit (73), whereas the fractional order $\sigma$ has the same significance as in Example 1, and the conformable order $\rho$ is responsible for speeding up or slowing down the voltage cycle.

Remark 8 If $\omega(\vartheta, \rho)=\vartheta^{1-\rho}$, then $h(\vartheta, 0, \rho)=\frac{\vartheta^{\rho}}{\rho}$, and the resulted solutions (72) and (76) recover the solutions obtained by the fractional conformable derivative in [18]. Moreover, 
the solutions obtained by the conformable and Caputo derivatives can be recovered if $\sigma=1$ and $\rho=1$, respectively.

\section{Conclusion}

We introduced a new family of fractional operators, called the IF operators. To define these fractional operators, we used a repeating procedure on general types of conformable integration and differentiation. The convolution kernels of the IF operators were given in conformable abstract forms. This broadens their scientific application scope compared to the traditional fractional operators. We have proved that the IF operators have features analogous to those of familiar fractional operators. Moreover, we suggested some specified function spaces to unveil the inverse and composition relations between the IF operators. Moreover, we noted that the fractional IF operators rely on two parameters so that, in modeling by these fractional operators, one of them is responsible for the memory trace, and the other for the speed up or slow down of the variation rate. Also, when these parameters approach specific values, the IF operators coincide with many fractional and conformable operators proposed earlier in the literature. As applications, we analyzed the resistor-capacitor electrical circuits in the framework of some IF operators. Hence new explicit formulas of the voltage were obtained in the cases of constant and periodic sources. Further, we displayed some graphical simulations to expound the overall effect of the IF operators on the voltage behavior. On the other hand, as we can see in Remarks $2-8$, our results extend and improve some results due to Jarad et al. [30, 31]. Therefore we conclude that the fractional left and right Caputo fractional derivatives, as well as the fractional left and right Caputo-Hadamard fractional derivatives, are particular cases of the Caputo LIFD and RIFD. Finally, To the author's awareness, the applications of the new IF operators do not limit to the electrical circuits, and they may have valuable applications in many scientific scopes.

\section{Appendix}

Let $\sigma, \rho \in \mathbb{C}$, where $\operatorname{Re}(\sigma), \operatorname{Re}(\rho)>0$. The biparameterized Mittag-Leffler function is defined as [19]

$$
E_{\sigma, \rho}(w)=\sum_{k=0}^{\infty} \frac{w^{k}}{\Gamma(k \sigma+\rho)} .
$$

If $\operatorname{Re}(\sigma)>0$ and $\operatorname{Re}(\rho)>0$, then the Laplace transform of $\vartheta^{\sigma-1} E_{\sigma, \rho}\left(\vartheta^{\sigma}\right)$ is given as

$$
\mathcal{L}\left(\vartheta^{\sigma-1} E_{\sigma, \rho}\left(\vartheta^{\sigma}\right)\right)(\varpi)=\frac{\varpi^{\sigma-\rho}}{\varpi^{\sigma}-1}, \quad \operatorname{Re}(\varpi)>1 .
$$

For $p \in \mathbb{N}$ and $m \in \mathbb{R}$, the $p$ th derivative of the function $E_{\sigma, \rho}\left(m \vartheta^{\sigma}\right)$ is given as

$$
E_{\sigma, \rho}^{(p)}\left(m \vartheta^{\sigma}\right)=\sum_{k=0}^{\infty} \frac{\Gamma(k+p+1)}{\Gamma(k+1)} \frac{m^{k} \vartheta^{k \sigma}}{\Gamma(k \sigma+p \sigma+\rho)} .
$$


Also, for $\operatorname{Re}(\sigma)>0, \rho \in \mathbb{R}$, and $\operatorname{Re}(\varpi)>|m|^{\frac{1}{\sigma}}$, the Laplace transform of $\vartheta^{p \sigma+\rho-1} E_{\sigma, \rho}^{(p)}\left(m \vartheta^{\sigma}\right)$ is given by

$$
\mathcal{L}\left(\vartheta^{p \sigma+\rho-1} E_{\sigma, \rho}^{(p)}\left(m \vartheta^{\sigma}\right)\right)=\frac{\Gamma(p+1) \varpi^{\sigma-\rho}}{\left(\varpi^{\sigma}-m\right)^{p+1}} .
$$

\section{Acknowledgements}

The authors extend their appreciation to the Deanship of Scientific Research at King Khalid University for funding this work through Research Groups Program under grant RGP.2/5/42.

\section{Funding}

This research was funded by King Khalid University, grant RGP.2/5/42.

\section{Abbreviations}

IF, Improved Fractional; LCD, Local Conformable Derivative; LGCD, Local Generalized Conformable Derivative; LGCDs, Local Generalized Conformable Derivatives; LIFI, Left Improved Fractional Integral; RIFI, Right Improved Fractional Integral; LIFD, Left Improved Fractional Derivative; RIFD, Right Improved Fractional Derivative; $\mathfrak{R} \mathfrak{C}$, Resistor-Capacitor.

\section{Availability of data and materials}

The data that support the findings of this study are available from the authors upon request.

\section{Competing interests}

The authors declare that they have no competing interests.

\section{Authors' contributions}

Both authors read and approved the final manuscript.

\section{Author details}

'Department of Mathematics, College of Science, King Khalid University, P.O. Box 9004, 61413, Abha, Saudi Arabia.

${ }^{2}$ Department of Engineering Mathematics and Physics, Faculty of Engineering, Al-Azhar University, 11371, Cairo, Egypt. ${ }^{3}$ Department of Mathematics, Faculty of Sciences, Al-Azhar University, 71524, Assiut, Egypt. ${ }^{4}$ Department of Computer

Science, College of Al Wajh, University of Tabuk, Tabuk, Saudi Arabia.

\section{Publisher's Note}

Springer Nature remains neutral with regard to jurisdictional claims in published maps and institutional affiliations.

Received: 25 March 2021 Accepted: 7 August 2021 Published online: 19 August 2021

\section{References}

1. Abdelhakim, A.A., Machado, J.A.T.: A critical analysis of the conformable derivative. Nonlinear Dyn. 95, 3063-3073 (2019)

2. Agarwal, P., Baleanu, D., Chen, Y.Q., Momani, S., Tenreiro Machado, J.A.: Fractional Calculus. Springer, Singapore (2019)

3. Alaria, A., Khan, A., Suthar, D.L., Kumar, D.: Application of fractional operators in modelling for charge carrier transport in amorphous semiconductor with multiple trapping. Int. J. Appl. Comput. Math. 5, 1-10 (2019)

4. Anastassiou, G.A.: Generalized Fractional Calculus. Springer, Cham (2021)

5. Atangana, A., Baleanu, D.: New fractional derivatives with non-local and non-singular kernel theory and application to heat transfer model. Therm. Sci. 20, 763-769 (2016)

6. Atangana, A., Goufo, E.F.D.: Cauchy problems with fractal-fractional operators and applications to groundwater dynamics. Fractals 28, 2040043 (2020)

7. Baleanu, D., Asad, J.H., Petras, I.: Numerical solution of the fractional Euler-Lagrange's equations of a thin elastic model. Nonlinear Dyn. 81, 97-102 (2015)

8. Baleanu, D., Asad, J.H., Petras, I., Elagan, S., Bilgen, A.: Fractional Euler-Lagrange equation of Caldirola-Kanai oscillator. Rom. Rep. Phys. 64, 1171-1177 (2012)

9. Baleanu, D., Petras, I., Asad, J.H., Velasco, M.P.: Fractional Pais-Uhlenbeck oscillator. Int. J. Theor. Phys. 51, 1253-1258 (2012)

10. Bhrawy, A.H., Ezz-Eldien, S.S.: A new Legendre operational technique for delay fractional optimal control problems. Calcolo 53, 521-543 (2016)

11. Boonsatit, N., Rajchakit, G., Sriraman, R., Lim, C.P., Agarwal, P.: Finite-/fixed-time synchronization of delayed Clifford-valued recurrent neural networks. Adv. Differ. Equ. 2021, 276 (2021)

12. Butzer, P.L., Kilbas, A.A., Trujillo, J.J.: Fractional calculus in the Mellin setting and Hadamard-type fractional integrals, J. Math. Anal. Appl. 269, 1-27 (2002)

13. Caputo, M., Fabrizio, M.: A new definition of fractional derivative without singular kernel. Prog. Fract. Differ. Appl. 1, $1-13(2015)$

14. Clauset, A., Shalizi, C.R., Newman, M.E.J.: Power-law distributions in empirical data. SIAM Rev. 51, 661-703 (2009)

15. Eslami, M., Rezazadeh, H., Rezazadeh, M., Mosavi, S.S.: Exact solutions to the space-time fractional Schrödinger-Hirota equation and the space-time modified KdV-Zakharov-Kuznetsov equation. Opt. Quantum Electron. 49, 279 (2017) 
16. Failla, G., Zingales, M.: Advanced materials modelling via fractional calculus: challenges and perspectives. Trans. R. Soc. A 378, 20200050 (2020)

17. Ghany, H.A., Hyder, A., Zakarya, M.: Exact solutions of stochastic fractional Korteweg de-Vries equation with conformable derivatives. Chin. Phys. B 29, 030203 (2020)

18. Gómez-Aguilara, J.F.: Fundamental solutions to electrical circuits of non-integer order via fractional derivatives with and without singular kernels. Eur. Phys. J. Plus 133, 197 (2018)

19. Gorenflo, R., Kilbas, A.A., Mainardi, F., Rogosin, S.V.: Mittag-Leffler Functions, Related Topics and Applications. Springer Monographs in Mathematics. Springer, Berlin (2014)

20. Gulgowski, J., Stefański, T.P., Trofimowicz, D.: On applications of elements modelled by fractional derivatives in circuit theory. Energies 13, 5768 (2020)

21. Habenom, H., Oli, A., Suthar, D.L.: $(p, q)$-Extended Struve function: fractional integrations and application to fractional kinetic equations. J. Math. 2021, 5536817 (2021)

22. Hammouch, Z., Mekkaoui, T., Agarwal, P.: Optical solitons for the Calogero-Bogoyavlenskii-Schiff equation in $(2+1)$ dimensions with time-fractional conformable derivative. Eur. Phys. J. Plus 133, 248 (2018)

23. Hyder, A.: White noise theory and general improved Kudryashov method for stochastic nonlinear evolution equations with conformable derivatives. Adv. Differ. Equ. 2020, 236 (2020)

24. Hyder, A.: The influence of the differential conformable operators through modern exact solutions of the double Schrödinger-Boussinesq system. Phys. Scr. 96, 115211 (2021)

25. Hyder, A., Soliman, A.H.: Exact solutions of space-time local fractal nonlinear evolution equations: a generalized conformable derivative approach. Results Phys. 17, 103135 (2020)

26. Hyder, A., Soliman, A.H.: A new generalized $\theta$-conformable calculus and its applications in mathematical physics. Phys. Scr. 96, 015208 (2021)

27. Hyder, A., Soliman, A.H.: An extended Kudryashov technique for solving stochastic nonlinear models with generalized conformable derivatives. Commun. Nonlinear Sci. Numer. Simul. 97, 105730 (2021)

28. Hyder, A., Soliman, A.H.: Analytical manner for abundant stochastic wave solutions of extended KdV equation with conformable differential operators. Math. Methods Appl. Sci., 1-13 (2021). https://doi.org/10.1002/mma.7317

29. Jain, S., Agarwal, R.P., Agarwal, P., Singh, P.: Certain unified integrals involving a multivariate Mittag-Leffler function. Axioms 10, 81 (2021)

30. Jarad, F., Abdeljawad, T., Baleanu, D.: On the generalized fractional derivatives and their Caputo modification. J. Nonlinear Sci. Appl. 10, 2607-2619 (2017)

31. Jarad, F., Uğurlu, E., Abdeljawad, T., Baleanu, D.: On a new class of fractional operators. Adv. Differ. Equ. 2017, 247 (2017)

32. Kar, R.: Optimal designs of analogue and digital fractional order filters for signal processing applications. CSI Trans. ICT 7, 175-180 (2019)

33. Katugampola, U.N.: New approach to a generalized fractional integral. Appl. Math. Comput. 218, 860-865 (2011)

34. Khalil, R., Al Horani, M., Yousef, A., Sababheh, M.: A new definition of fractional derivative. J. Comput. Appl. Math. 264, 65-70 (2014)

35. Khater, M.M.A., Baleanu, D.: On abundant new solutions of two fractional complex models. Adv. Differ. Equ. 2020, 268 (2020)

36. Kilbas, A.A.: Hadamard type fractional calculus. J. Korean Math. Soc. 38, 1191-1204 (2001)

37. Lazopoulos, K.A., Lazopoulos, A.K.: Fractional vector calculus and fluid mechanics. J. Mech. Behav. Mater. 26, 43-54 (2017)

38. Mahata, S., Saha, S.K., Kar, R., Mandal, D.: Optimal design of fractional order low pass Butterworth filter with accurate magnitude response. Digit. Signal Process. 72, 96-114 (2018)

39. Martínez, L., Rosales, J.J., Carreño, C.A., Lozano, J.M.: Electrical circuits described by fractional conformable derivative. Int. J. Circuit Theory Appl. 46, 1091-1100 (2018)

40. Meng, X., Nane, E.: Space-time fractional stochastic partial differential equations with Lévy noise. Fract. Calc. Appl. Anal. 23, 224-249 (2020)

41. Padula, F., Visioli, A.: Advances in Robust Fractional Control. Springer, Cham (2015)

42. Penga, Y., Zhaoa, J., Sepehrnoori, K., Li, Z.: Fractional model for simulating the viscoelastic behavior of artificial fracture in shale gas. Eng. Fract. Mech. 228, 106892 (2020)

43. Pezza, L., Pitolli, F.: A multiscale collocation method for fractional differential problems. Math. Comput. Simul. 147, 210-219 (2018)

44. Podlubny, l.: Fractional Differential Equations. Academic Press, San Diego (1998)

45. Rezazadeh, H., Khodadad, F.S., Manafian, J.: New structure for exact solutions of nonlinear time fractional Sharma-Tasso-Olver equation via conformable fractional derivative. Appl. Appl. Math. 12, 405-414 (2017)

46. Sene, N., Gómez-Aguilar, J.F.: Analytical solutions of electrical circuits considering certain generalized fractional derivatives. Eur. Phys. J. Plus 134, 260 (2019)

47. Soliman, A.H., Hyder, A.: Closed-form solutions of stochastic KdV equation with generalized conformable derivatives. Phys. Scr. 95, 065219 (2020)

48. Sunarto, A., Agarwal, P., Sulaiman, J., Chew, J.V.L., Aruchunan, E.: Iterative method for solving one-dimensional fractional mathematical physics model via quarter-sweep and PAOR. Adv. Differ. Equ. 2021, 147 (2021)

49. Suthar, D.L., Agarwal, P., Amsalu, H.: Marichev-Saigo-Maeda fractional integral operators involving the product of generalized Bessel-Maitland functions. Bol. Soc. Parana. Mat. 39, 95-105 (2021)

50. Tenreiro Machado, J.A., Lopes, A.M.: Relative fractional dynamics of stock markets. Nonlinear Dyn. 86, 1613-1619 (2016)

51. Tenreiro Machado, J.A., Mata, M.E.: A fractional perspective to the bond graph modelling of world economies. Nonlinear Dyn. 80, 1839-1852 (2015)

52. Uchaikin, V.V.: Fractional Derivatives for Physicists and Engineers. Springer, Berlin (2013)

53. Yang, X.J., Gao, F., Ju, Y.: General Fractional Derivatives with Applications in Viscoelasticity. Academic Press, London (2020)

54. Zeng, S., Cen, J., Atangana, A., Nguyen, V.T.: Qualitative analysis of solutions of obstacle elliptic inclusion problem with fractional Laplacian. Z. Angew. Math. Phys. 72, 30 (2021) 
55. Zhao, D., Luo, M.: General conformable fractional derivative and its physical interpretation. Calcolo 54, 903-917 (2017)

56. Zhao, D., Pan, X., Luo, M.: A new framework for multivariate general conformable fractional calculus and potential applications. Physica A 510, 271-280 (2018)

Submit your manuscript to a SpringerOpen ${ }^{\circ}$ journal and benefit from:

- Convenient online submission

- Rigorous peer review

- Open access: articles freely available online

- High visibility within the field

- Retaining the copyright to your article

Submit your next manuscript at $\boldsymbol{\Delta}$ springeropen.com 